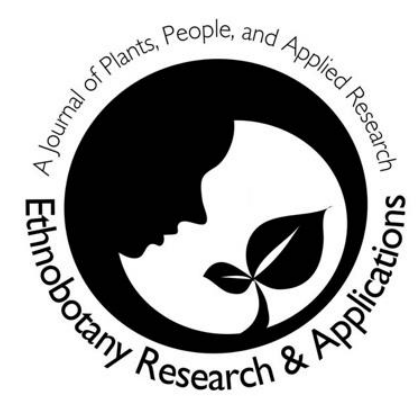

\title{
The indigenous uses of plants from Siberut, Mentawai, Indonesia
}

Samantha M. Lee ${ }^{1,2^{*}}$, J. Doland Nichols ${ }^{1}$, David Lloyd ${ }^{1}$, Susanti Sagari3, Filemon Sagulu3, Iskandar Z. Siregar ${ }^{4}$, Adisti Permatasari Putri Hartoyo ${ }^{4}$, Rob Henry ${ }^{2}$, Nurainas ${ }^{5}$

\author{
Correspondence \\ Samantha M. Lee ${ }^{1,2^{*}}$, J. Doland Nichols ${ }^{1}$, David Lloyd ${ }^{1}$, Susanti Sagari ${ }^{3}$, Filemon Sagulu ${ }^{3}$, Iskandar Z. Siregar ${ }^{4}$, \\ Adisti Permatasari Putri Hartoyo ${ }^{4}$, Rob Henry ${ }^{2}$, Nurainas ${ }^{5}$ \\ ${ }^{1}$ Forest Research Centre, Southern Cross University, Lismore, Australia \\ 2 Indigenous Education Foundation, Melbourne, Australia \\ ${ }^{3}$ Yayasan Pendidikan Budaya Mentawai, Muntei, Siberut, Mentawai, Indonesia \\ 4 Department of Silviculture, Faculty of Forestry, Institut Pertanian Bogor, Bogor, Java, Indonesia \\ ${ }^{5}$ Herbarium Universitas Andalas, Universitas Andalas, Padang, West Sumatra, Indonesia \\ *Corresponding Author: samantha.lee@scu.edu.au
}

Ethnobotany Research \& Applications 22:18 (2021)

\section{Research}

\begin{abstract}
Background. Indigenous knowledge systems, like those traditionally practiced by the Mentawai people from the Mentawai Islands, West Sumatra, Indonesia, are a deep-time laboratory that examines how people manage or failed to find adaptive solutions to changes in their surroundings, including biodiversity loss and changing climate. However, many of these practices, including Mentawai's traditional knowledge system, arat sabulungan, are eroding. Given that Indigenous knowledge systems are believed to make up at least half of humanity's intellectual heritage, adequate documentation of traditional ecological knowledge is needed, as too are appropriate systems that support the perpetuation of such knowledge. This paper identifies the indigenous uses of Siberut's flora and explores how this knowledge may underlie the conservation of Siberut's unique biodiversity. Mentawai values and attitudes toward their traditional ecological knowledge and reasons why their traditional knowledge is eroding are also examined.
\end{abstract}

Methods: Primary data was derived from various sources, including: observation of peoples' day-to-day activities; community surveys (number of participants $=494$ ) and key informant interviews (number of informants $=21$ ); and plant collection and identification. Research participants in the community survey were chosen based on their interest and willingness to partake in the survey. Key informants were chosen based on their reputation within the community on the extent of their plant knowledge.

Results: Ethnobotanical data suggests that the Mentawai have ethnobotanical uses for at least half of all plant species on record. Though 98 percent of respondents felt their traditional knowledge is important for the future of Mentawai, a majority felt there were substantial threats to the continuation of their traditions.

Conclusion: Data gathered during the research period suggests that Mentawai indigenous practices and traditions play a critical role in conserving Siberut's unique biodiversity.

Key words: Biodiversity; Indigenous peoples; traditional ecological knowledge; Mentawai Islands; Indonesia; tropical conservation; Mentawai people 


\section{Background}

\section{Indigenous knowledge systems}

Indigenous peoples inhabit some of the most biologically diverse regions globally and add substantially to the world's cultural, linguistic and intellectual heritage (Davis 2009, Gray 1991, Posey 1999, UNDESA 2009). Accumulated over millennia, traditional ecological knowledge (TEK) spans multiple dimensions and scales, and is verified through repetition, empirical observations and recognition of patterns (Mazzocchi 2006, Nicholas 2018). Moreover, these knowledge systems are often: collectively owned; have co-evolved with ecosystems; integrate benefits across many dimensions, and usually pre-date Western science (Mazzocchi 2006, Tiwari et al. 2017, UNDESA 2009, Wilder et al. 2016). Yet concerns remain in relation to the conservation of Indigenous peoples' knowledge systems as they continue to be marginalised, expropriated and disparaged; forced to adhere to foreign education, religion and politics (UNDESA 2009). Moreover, those processes that threaten biological diversity and ecosystem health also impact indigenous populations (Sheil et al. 2005), causing detrimental effects to their physical and mental health and wellbeing, and their traditional lands and biomes (Maffi 2002, Minority Rights Groups International 2017, Persoon 2008). Yet, Indigenous peoples, whose values, systems and stewardship regularly interact with, sustain, and often perpetuate or enrich traditional lands and ecosystems, offer significant contributions to global biodiversity conservation efforts (Berkes et al. 2000, Corrigan et al. 2018, Corrigan \& HayEdie 2013, Posey 1999, Sheil \& Lawrence 2004, Sobrevila 2008, Standley \& Hill 2011, Sujarwo \& Caneva 2016, Wilder et al. 2016). Given the current global crisis - namely, ecological degeneration, the possibility of climate collapse and unprecedented biodiversity loss, adequate documentation of TEK is needed, as too are appropriate systems that support the perpetuation of such knowledge.

Mentawai, West Sumatra, Indonesia, is home to a rich, biologically diverse rainforest and unique hunter-gatherer culture. Purportedly, the Mentawai people have occupied these islands for thousands of years, maintaining a strong cultural and spiritual connection with the forest they inhabit (Schefold 1989). In varying degrees, the Mentawai people still practice arat sabulungan, a complex, animistic belief system based on the sacred relationships between themselves and the spirits that reside in all animate and inanimate objects. These practices are based on the overarching priority to live in harmonious balance with nature, and offer the Mentawai people a subsistence lifestyle that can provide all food, resources and medicines from the forests surrounding the uma (traditional clan houses usually built on river tributaries throughout the forest). Tribal elders, or sikerei/ sikebbu'kat, are the primary keepers of this knowledge and work to maintain the health of their community, acting as shaman, educator and healer.

Since 1954, foreign practice and ideology were introduced and enforced throughout Mentawai as part of a "modernisation program" for isolated communities (Colfer \& Resosudarmo 2002). Labelled as 'backward' and 'primitive', the Mentawai people were removed from their ancestral lands and relocated into 'modern' resettlement villages, where traditional agriculture, religion, education and artefacts were forbidden. As foreign developments and a cash-based economy have become established within the villages; as more children are being sent to government-run schools; as more villagers become reliant on imported foods rather than forest foods and traditional agricultural provisions; and, as fewer youth are undertaking apprenticeships to become sikerei, the transmission of Mentawai TEK is hindered, and more quickly becoming diluted (Bakker 1999, Kusakabe et al. 2015, van Klooster et al. 2019, Wilder et al. 2016). Despite national policies softening to accommodate traditional beliefs and practices (Boedhihartono 2017), 99 percent of the Mentawai population live within the resettlement villages, with very limited or no access to their traditional knowledge and practices (Persoon 1998, Persoon 2008). The gap between the younger generation and the TEK held and taught by the sikerei and sikebbu'kat (respected elders) is substantial, and growing rapidly.

\section{Aims of this study}

The continued richness of biodiversity, intact ecosystems and perpetuation of Mentawai TEK is assumed to be intrinsic to Mentawai wellbeing, and ultimately fundamental to their communities' survival. Additionally, it is believed that arat sabulungan - Mentawai TEK - governs and, in many ways, may perpetuate Mentawai's biodiversity (Berkes et al. 2000, Colfer \& Resosudarmo 2002, Reyes-García et al. 2014). Considering the Mentawai community's values, agenda, and long-term goals, this study examines Mentawai uses of plants and the associated traditions. This study is the first to rank these plants according to their perceived importance by the community, and thus offers a fuller understanding of Mentawai TEK practices, customs and taboos, and local conservation priorities. Collaboration with an Indigenous-led non-profit organisation and their research team was foundational to the research approach as it helped us gain greater depth, accuracy, and relevance of results and ramifications. 


\section{Materials and methods}

\section{Study area}

Maileppet ( $\left.1^{\circ} 33^{\prime} 14 \mathrm{~S}, 99^{\circ} 10^{\prime} 58^{\prime \prime} \mathrm{E}\right)$ is a coastal village approximately 5 kilometres north of the main port, Muarasiberut, in Southern Siberut, West Sumatra, Indonesia (Figure 1). Nearby Muntei village $\left(1^{\circ} 34^{\prime} 56 \mathrm{~S}, 99^{\circ} 11^{\prime} 25\right.$ E) is situated west of Maileppet on the Muntei River (see Figure 1). Both village districts predominately speak the Sabirut dialect of the Mentawai language. Surrounding the villages are disturbed secondary forests predominately covered by Metroxylon sagu Rottb. (Arecaceae), Musa L. (Musaceae), Durio Adans. (Malvaceae), Cocos nucifera L. (Arecaceae), and other useful or commercial crops (e.g., Areca catechu L. (Arecaceae) and Hevea brasiliensis (Willd. ex A.Juss.) Müll.Arg (Euphorbiaceae)), with mangrove forests lining the coast, and Colocasia esculenta (L.) Schott (Araceae). In addition, pugette'an (traditional taro garden fields) and mone (clan-owned growing fields) are scattered throughout the landscape. Maileppet and Muntei village regions are located within Siberut's development zone, where forestry, agricultural and urban developments may occur, and are not within the Siberut National Park conservation zone (WWF 1980).

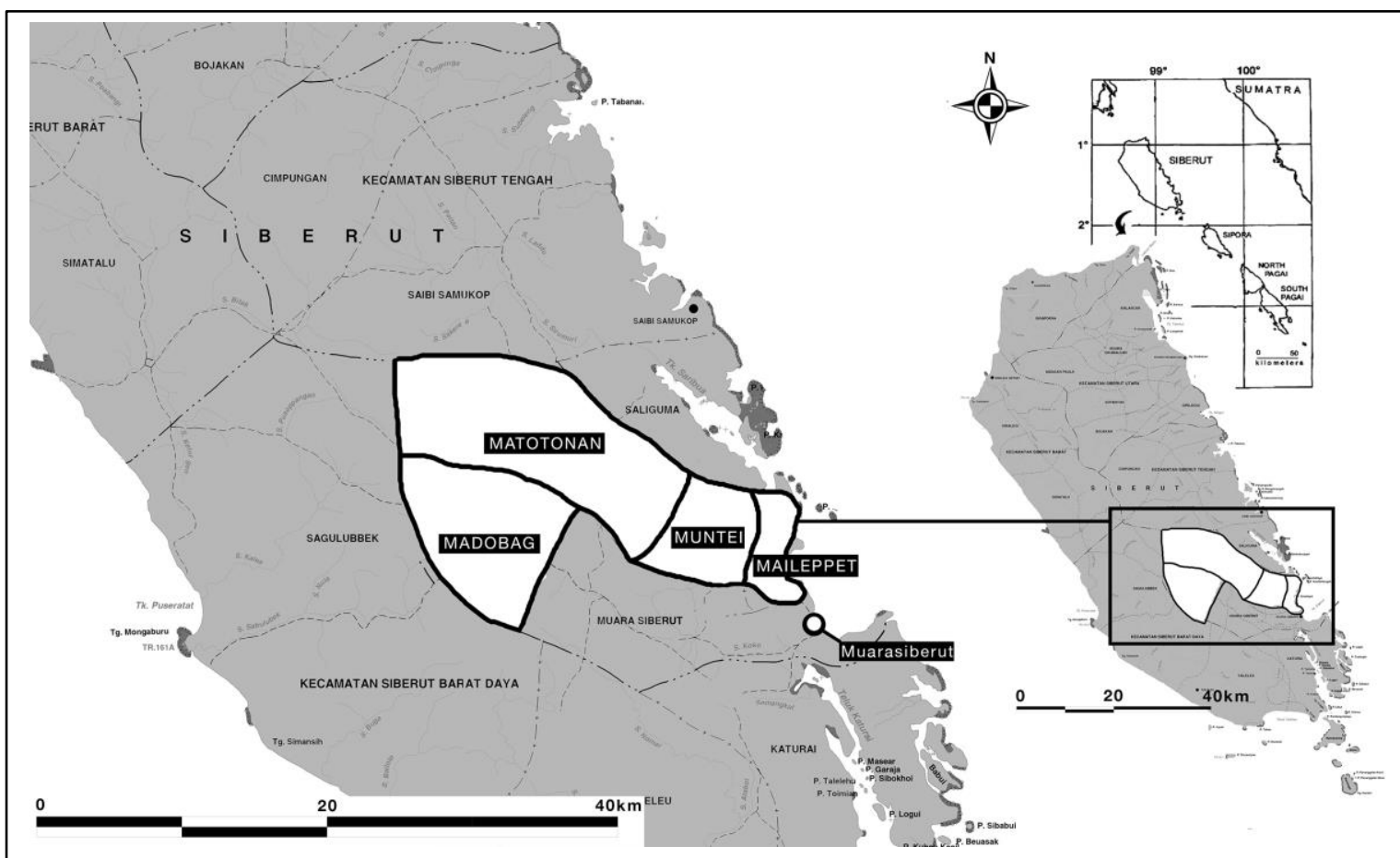

Figure 1. Map of study area showing locations of Muntei, Maileppet, Matotonan and Madobag village districts on Siberut Island, Mentawai, West Sumatra, Indonesia (Map source: Kebupaten Kepulauan Mentawai \& PT Dantarsa Gatra 2004; Whitten 1982).

The Madobag and Matotonan regions, $1^{\circ} 35^{\prime} 43 \mathrm{~S}, 99^{\circ} 5^{\prime} 2 \mathrm{E}$ and $1^{\circ} 32^{\prime} 19 \mathrm{~S}, 99^{\circ} 0^{\prime} 58 \mathrm{E}$ respectively, are situated along the Sarereiket River, a Muntei River tributary, approximately 20-25 kilometres upriver northwest from Muarasiberut (see Figure 1). Both districts share the Rereiket dialect of the Mentawai language. The region is remote and usually only accessible by pompong (small dugout canoe with a 2 HP engine) or foot. The surrounding vegetation mixes closed-canopy forests, pumonean (food forests), pugette'an, and sagu (Metroxylon sagu) swamps. The Madobag and Matotonan village regions fall within the Siberut National Park traditional use buffer zone, where all traditional use and activities may take place, and commercial logging is not permitted (WWF 1980).

\section{Data Collection}

This project was carried out in collaboration with researchers from Yayasan Pendidikan Budaya Mentawai (YPBM), a grassroots non-profit organisation providing cultural and ecological knowledge learning opportunities for students living in resettlement villages in Mentawai. Through a participatory approach, the study sought to empower YPBM collaborators by facilitating research training activities that might enhance team members' existing skills so that plant identification and ethnobotanical documentation, and monitoring and evaluation activities may be continued following the completion of this study. 
The lead researcher lived with the Mentawai community from January to December 2019, and was mostly based in Muntei village to facilitate daily collaboration with YPBM at their office base. Several field trips to the Madobag and Matotonan districts were undertaken during this time. In addition, the lead researcher frequently accompanied sikerei and sikebbu'kat whilst they were collecting or preparing medicines. Researcher involvement in day-to-day household and wider community activities allowed more accurate detection of community needs, wants and attitudes, and enabled the lead researcher to become familiar with Mentawai plant traditions and practices in context (similar to experiences of Nolan \& Turner 2011). This involvement also supported the detection of emerging socio-cultural trends (Alexiades 1996). Documentation activities (including photography and videography) focused on capturing the value and relevance of TEK to the Mentawai people, whilst also recording individual plant specimens and uses. Voucher specimens for the plants identified as the most useful to the Mentawai people were collected according to standards outlined in Cunningham (2001) and Martin (1995). Specimens were identified using existing literature and deposited in the herbarium at Universitas Andalas (ANDA) for identification and storage [See Appendix 1 for collection details]. These specimens were still awaiting cataloguing at ANDA at the time of publication.

Semi-structured interviews were carried out on a one-on-one basis by Mentawai collaborators from February to March 2019. A total of 494 participants were surveyed. Research participants were chosen based on their interest and willingness to partake in the survey. Recognising that most Mentawai community members hold some level of botanical knowledge (Nolan \& Turner, 2011), and to elucidate differences in opinion and knowledge levels between population groups, no bias was given for age, ethnicity, level of education or occupation for those participating in the community survey. Nearly half of all respondents were 18-40 years of age, nearly one-third were over 41 years of age and 12 percent were aged between 12-17 years of age. Fifty-seven percent of participants were male; 43 percent were female. One-quarter of all respondents had not completed any formal schooling, one third had only completed primary school, 38 percent had completed middle and/or high school, and 5 percent of participants had attended university. Sixty-eight percent of respondents identified their religion as Catholic, 26 percent Islam, and 6 percent Protestant.

As suggested by Mentawai collaborators, the lead researcher did not interview participants in the community survey to prevent participants from becoming shy or distracted due to language barriers. The community survey had two sections: i) a mostly closed-ended questionnaire about individual perceptions of Mentawai TEK, and ii) a 'freelisting' activity, where respondents were asked to cite useful 'ethnospecies' (plant specimens identified as individual species according to Mentawai informants) within previously defined categories, namely: food, medicine, construction, ceremonial, firewood, tools/handicrafts) (Quinlan 2005). No limit was given to how many plants each respondent could list for each category. From these lists, a freelisting matrix was created to document the relative frequency of citation (RFC), and the average order in which each plant was listed, thus attempting to determine the overall cultural saliency of each ethnospecies, where:

$$
R F C=(\text { number of times an ethnospecies was cited }) /(\text { total number of informants }) .
$$

The freelisting matrix also sought to reveal variations between age, gender and regional demographics (Caruso 2015, Quinlan 2005, Vogl et al. 2004). Language variations were identified when possible for each ethnospecies cited. Respondent answers that cited plant parts (e.g. loina', kayu (Bahasa Indonesia), timber), names of ceremonies (e.g. puliaijat pangurei), or objects (e.g., opa, jaraik), and not plant species were excluded from the analysis.

To clarify results gathered from the community survey, 21 key informant interviews with sikerei and sikebbu'kat were conducted. Key informants were selected based on community consensus of these participants being perceived as being highly knowledgeable and experienced in Mentawai plant knowledge. Interviews were carried out by YPBM team members who were fluent in the Sabirut and Rereiket dialects. Using results from the freelisting activity described above, key informant interview questions focused on refining the uses and descriptions of plant data extracted from the community surveys, and confirming that data gathered from these surveys adequately reflected the perceived usefulness and value of species within Mentawai plant traditions. Open questions focused on elucidating how participants gained their knowledge, whether they had taught or were teaching anyone else, and whether they believed the ways that knowledge is being shared and practiced may be changing, under threat, or eroding. 
Data analysis

The cultural significance of each ethnospecies was calculated as specified by Silva et al. (2006) as:

$$
C S I=\sum(i \times e \times c) \times C F .
$$

Where $i=$ ethnospecies management [wild (1) or cultivated (2)]; $e=$ use preference [not preferred (1) or preferred (2)]; $c$ = use frequency [rarely (1), sometimes (1.5) frequently (2) used]; CF = correction factor [number of citations for a given ethnospecies divided by the number of citations for the most-mentioned species].

To measure the proportion of informants who regard an ethnospecies as most important, the Importance Value (IV) formula, adapted by Byg \& Balslev (2001) from Phillips \& Gentry (1993), was used:

$$
I V_{s}=n_{i s} / n
$$

Where $n_{i s}=$ the number of informants who consider ethnospecies $s$ most important; and $n=$ the total number of informants.

\section{Results}

\section{Overview}

A total of 452 ethnospecies were recorded during the initial 10-month documentation period (January to October 2019): 339 ethnospecies were spontaneously listed during freelisting activities (number of informants $=494$ ) and, 127 ethnospecies cited in key informant interviews $(n=21)$. When asked to clarify whether any species cited may have two or more varieties, informants were able to cite an additional 166 varieties (e.g., aileleppet includes aileleppet simaingo, aileleppet simabulau, aileleppet kaeru'), including 17 varieties of mago' (Musa spp.), 14 varieties of gette' (Colocasia esculenta), and 13 rattan types. A total of 225 ethnospecies could be identified to genus or family level, from which 83 botanical families were represented. Zingiberaceae (20 ethnospecies), Poaceae (12 ethnospecies) and Araceae (11 ethnospecies) were the most highly represented families (Figure 2). Most plant species are believed to be native to the Mentawai Islands.

\section{Frequently cited plants in the community freelisting activity}

The average number of ethnospecies listed by respondents during the community freelisting activities was 21.8 ( \pm 12.76 SD, $\pm 0.57 \mathrm{SE}$ ) (Figure 3). Gender did not play a significant role in determining the number of plants cited by respondents ( $t$-test, $p>0.05$ ). However, there was a correlation between age and the average number of plants cited by respondents (ANOVA single factor test, $p<0.05$ ), with men over 60 years of age able to cite significantly more species $(M=28.1, \pm 13.3 \mathrm{SD}, \pm 0.73 \mathrm{SE}$ ) than younger respondents (Figure 4). Of the total number of useful ethnospecies cited, 39 percent could be used medicinally, 30 percent used for construction, 25 percent were used for firewood, 24 percent had ceremonial purposes, 19 percent were edible, and 14 percent used for tool-making. One-third of all ethnospecies were listed in more than one category. Of the 339 ethnospecies cited during the community freelisting activities, 95 ethnospecies were mentioned by only one respondent.

A total of 64 ethnospecies were listed as food sources. Sagu (Metroxylon sagu), gette' (Colocasia esculenta) and mago' (Musa spp.) were most frequently mentioned as the most important food sources (Table 1). Fifty-seven ethnospecies cited as food sources were mentioned by fewer than 20 percent of respondents. A total of 134 ethnospecies were cited for medicinal purposes. Aileleppet (Graptophyllum pictum (L.) Griff. (Acanthaceae)), kakainau' (Hedychium coronarium J.Koenig (Zingiberaceae) / H. flavescens Carey ex Roscoe (Zingiberaceae)), talingengeng (Piper betle L. (Piperaceae)), kopuk (Kaempferia galanga L. (Zingiberaceae)), and mumumen (Mussaenda frondosa L. (Rubiaceae)) were ranked highly, and were mentioned by more than 20 percent of respondents. Only one respondent mentioned thirty-nine medicinal ethnospecies. Eighty-five ethnospecies were listed as important ceremonial plants, of which only five were listed by more than 20 percent of respondents. Medicinal plants were listed highly for ceremonial purposes, along with important traditional foods such as sagu, gette', toitet, and decorative plants sura' (Codiaeum variegatum (L.) Rumph. ex A.Juss. (Euphorbiaceae)), bekeu (Hibiscus rosa-sinensis L. (Malvaceae)), katsaila (Arenga pinnata (Wurmb.) Merr. (Arecaceae)). A total of 103 ethnospecies were considered as important building materials. Only one respondent cited twenty-four ethnospecies. Katuka (Shorea pauciflora King (Dipterocarpaceae)), sagu, kakatdut (Alstonia spatulata Blume (Apocynaceae)), mancemi (Hopea dryobalanoides Miq. (Dipterocarpaceae) and manggeak (bamboo types) were 
most frequently cited as important building materials. Respondents from the Sarereiket region also ranked kaboi (Pentace triptera Mast. (Malvaceae)) highly for building purposes.

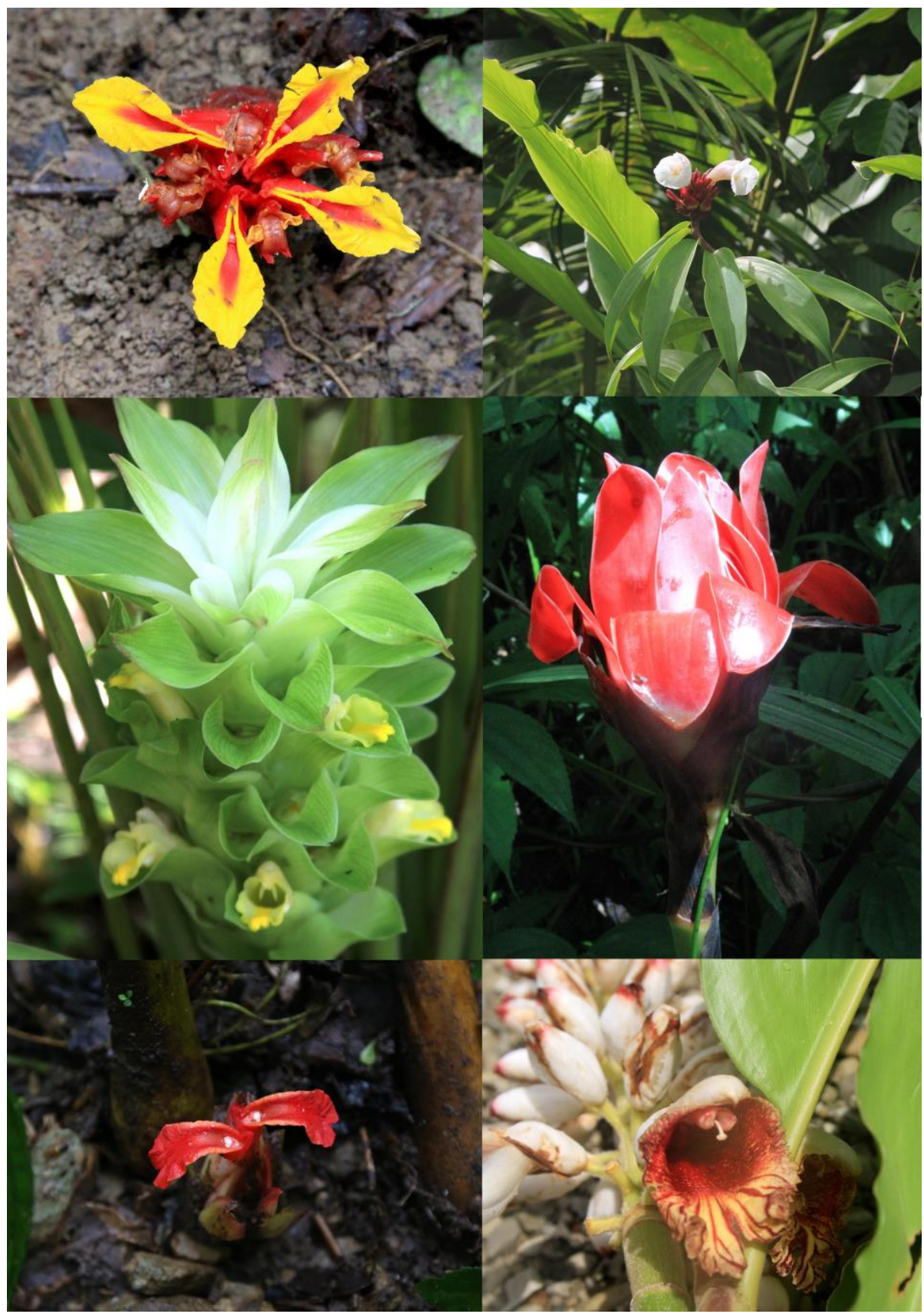

Figure 2. Six examples of important Zingiberales plant species found in the Sabirut and Sarereiket regions of Siberut, Mentawai Islands, Indonesia. From left to right: pelekak (Etlingera coccinea (Blume) S.Sakai \& Nagam. (Zingiberaceae)), bagli-bagli (Hellenia speciosa (J.Koenig) S.R.Dutta (Costaceae) kiniu (Curcuma longa L. (Zingiberaceae)), Tottot mamai (Sabirut dialect) / Gojo (Rereiket dialect) (Etlingera elatior (Jack) R.M.Sm (Zingiberaceae)), kukuet (Hornstedtia conica Ridl. (Zingiberaceae)), and kojo koddiai (Siberut dialect) / kasisingin (Sabirut dialect) / pasisingin (Rereiket dialect) (Alpinia malaccensis C.Presl (Zingiberaceae)).

A total of 86 ethnospecies were listed as firewood, with three ethnospecies cited by 20 percent of respondents. Some respondents commented that all wood might be used as firewood provided they were dry. The most frequently cited ethnospecies for firewood were manggeak, sagu, and sibetu (Sabirut dialect) / eru'teinung (Rereiket dialect) (Melastoma sylvaticum Blume (Melastomataceae)). For handicraft and tool-making, a total of 46 ethnospecies were cited. Rattan kinds, including pelege (Calamus sp. L (Arecaceae)), mandorou'(Calamus caesius 
Blume (Arecaceae)), and sasa (Calamus sp.), were ranked most highly, along with osap (Gleicheniaceae C.Presl), toitet, manggeak and sagu.

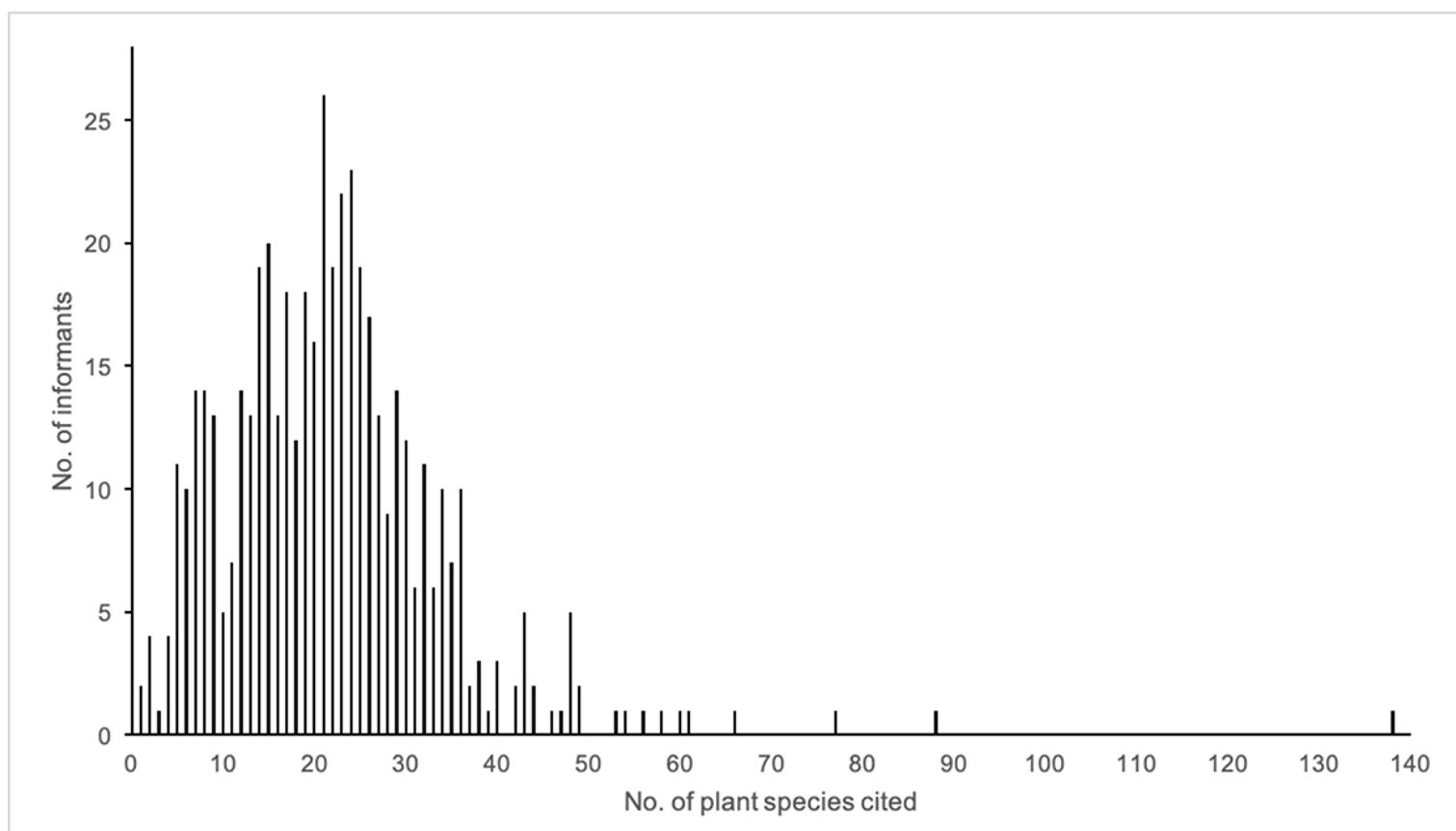

Figure 3. Distribution of plant knowledge among the Mentawai people living in the Sabirut and Sarereiket regions of Siberut, Mentawai Islands, as indicated by the list length of informants $(n=494)$ who cited certain plants across all surveyed categories. Total number of plant species mentioned as being useful was 339. Average list length was $21.8( \pm 12.76 \mathrm{SD}, \pm 0.57 \mathrm{SE})$.

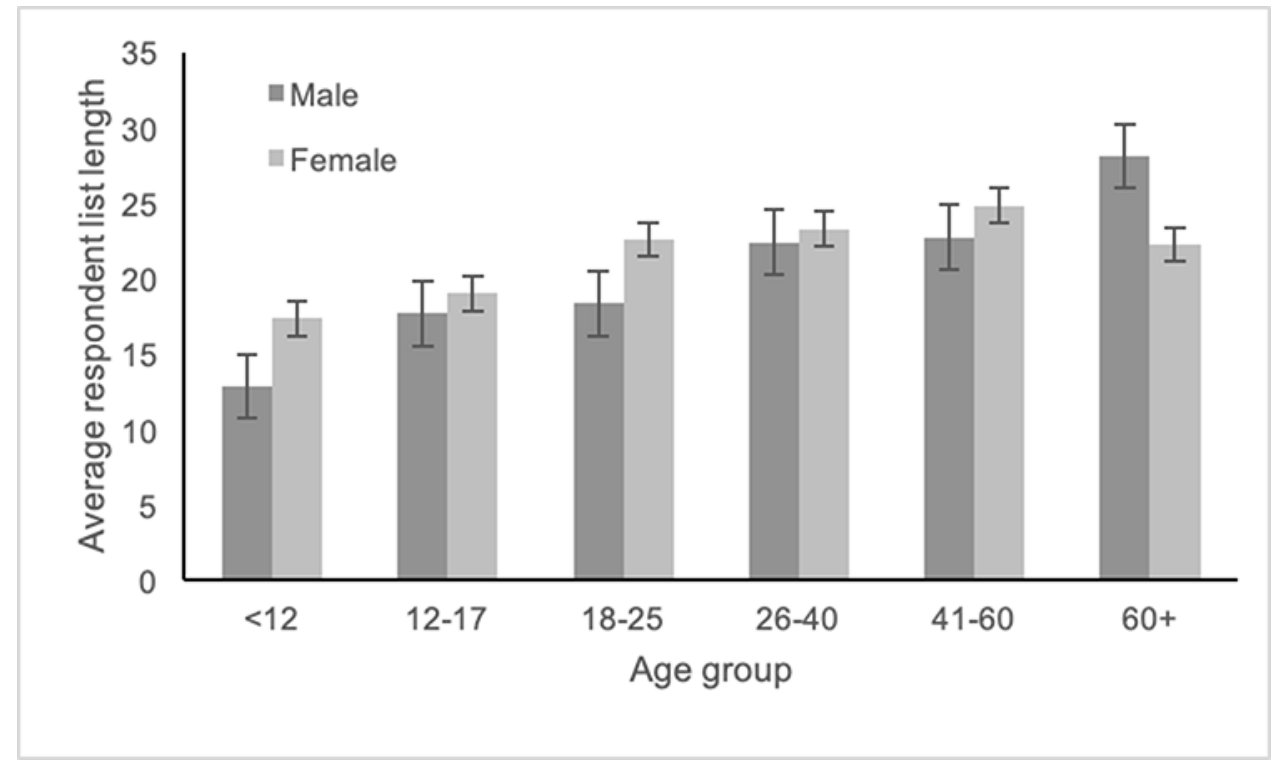

Figure 4. Average number of plants cited during freelisting surveying per age category and gender by the Mentawai people living in the Sabirut and Sarereiket regions of Siberut, Mentawai Islands $(n=494)$. Men over 60 years of age cited more plants than other age and gender categories (mean 28.1, \pm 16.14 SD, \pm 0.72 SE). Overall, there was no significance difference among list length between genders (ANOVA single factor test, $p$ $<0.05)$. 
Table 1. The top five most frequently cited ethnospecies within each category (food / nutritional, medicinal, ceremonial, building, firewood, and tool-making / handicraft) by the Mentawai people living in the Sabirut and Sarereiket regions of Siberut, Mentawai Islands, Indonesia. Relative frequency of citation (RFC) shows the proportion of the population who cited that ethnospecies within that category, and average rank (Av. rank) is the average list order in which respondents cited that ethnospecies for that category. Sagai (Sab) / Sagu (Sar) (Metroxylon sagu Rottb. (Arecaceae)) was frequency cited as important for all six categories. (Ment -Mentawai language, Sib -Siberut dialect, Sab - Sabirut dialect, Sar -Rereiket dialect, BI - Bahasa Indonesia).

\begin{tabular}{|c|c|c|c|c|}
\hline Family & Scientific name & Vernacular name/s & RFC & Av. rank \\
\hline \multicolumn{5}{|l|}{ Food / nutritional } \\
\hline Arecaceae & Metroxylon sagu Rottb. & Sagai (Sab) / Sagu (Sar) & 0.96 & 1.4 \\
\hline Araceae & Colocasia esculenta (L.) Schott & Gette' (Ment) / Keladi (BI) & 0.78 & 2.6 \\
\hline Musaceae & Musa spp. L. & Bago' (Sab) / Mago' (Sar) / Pisang (BI) & 0.76 & 2.7 \\
\hline Arecaceae & Cocos nucifera L. & Toitet (Ment) / Kelapa (BI) & 0.47 & 3.9 \\
\hline Malvaceae & Durio spp. Adans. & Doriat (Ment) / Durian (BI) & 0.21 & 5.2 \\
\hline \multicolumn{5}{|l|}{ Medicinal } \\
\hline Acanthaceae & Graptophyllum pictum (L.) Griff. & Aileleppet (Ment) & 0.44 & 2.7 \\
\hline Zingiberaceae & $\begin{array}{l}\text { Hedychium coronarium } \\
\text { J.Koenig / Hedychium } \\
\text { flavescens Carey ex Roscoe }\end{array}$ & Kakainau' (Sab) / Simakainau' (Sar) & 0.43 & 2.7 \\
\hline Piperaceae & Piper betle L. & Talingengeng (Ment) / Sirih (BI) & 0.33 & 2.6 \\
\hline Zingiberaceae & Kaempferia galanga L. & $\begin{array}{l}\text { Kopuk (Sab) / Sikopuk (Sar) / Kencur } \\
(\mathrm{BI})\end{array}$ & 0.32 & 3.4 \\
\hline Rubiaceae & Mussaenda frondosa $\mathrm{L}$. & Mumunen (Sib) & 0.22 & 2.6 \\
\hline \multicolumn{5}{|l|}{ Ceremonial } \\
\hline Euphorbiaceae & $\begin{array}{l}\text { Codiaeum variegatum (L.) } \\
\text { Rumph. ex A.Juss. }\end{array}$ & Sura' (Ment) & 0.36 & 1.9 \\
\hline Arecaceae & Metroxylon sagu Rottb. & Sagai (Sab) / Sagu (Sar) & 0.24 & 2.2 \\
\hline Acanthaceae & Graptophyllum pictum (L.) Griff. & Aileleppet (Ment) & 0.24 & 3.2 \\
\hline Araceae & Colocasia esculenta (L.) Schott & Gette' (Ment) / Keladi (BI) & 0.22 & 2.5 \\
\hline Zingiberaceae & $\begin{array}{l}\text { Hedychium coronarium } \\
\text { J.Koenig / Hedychium } \\
\text { flavescens Carey ex Roscoe }\end{array}$ & Kakainau' (Sab) / Simakainau' (Sar) & 0.21 & 3.2 \\
\hline \multicolumn{5}{|l|}{ Building } \\
\hline Dipterocarpaceae & Shorea pauciflora King & Katuka (Ment) & 0.50 & 1.7 \\
\hline Arecaceae & Metroxylon sagu Rottb. & Sagai (Sab) / Sagu (Sar) & 0.34 & 2.7 \\
\hline Apocynaceae & Alstonia spatulata Blume & Kakatdut (Sab) / Gite (Sar) / Pulai (BI) & 0.29 & 2.8 \\
\hline Dipterocarpaceae & Hopea dryobalanoides Miq. & Mancemi (Ment) & 0.28 & 2.8 \\
\hline Poaceae & Bamboo types & $\begin{array}{l}\text { Manggeak (Ment) / Obbuk (Ment) / } \\
\text { Bambu (BI) }\end{array}$ & 0.27 & 3.0 \\
\hline \multicolumn{5}{|l|}{ Firewood } \\
\hline Poaceae & Bamboo types & $\begin{array}{l}\text { Manggeak (Ment) / Obbuk (Ment) / } \\
\text { Bambu (BI) }\end{array}$ & 0.45 & 2.3 \\
\hline Arecaceae & Metroxylon sagu Rottb. & Sagai (Sab) / Sagu (Sar) & 0.31 & 2.5 \\
\hline Melastomataceae & Melastoma sylvaticum Schltdl. & Sibetu (Sab) / Eru'teinung (Sar) & 0.20 & 2.9 \\
\hline Euphorbiaceae & Macaranga sp. Thouars & Katumanggei (Ment) & 0.18 & 2.6 \\
\hline Rhizophoraceae & Rhizophora apiculata Blume & Bakat (Ment) / Bakau (BI) & 0.18 & 2.0 \\
\hline \multicolumn{5}{|l|}{$\begin{array}{l}\text { Tool-making / } \\
\text { handicrafts }\end{array}$} \\
\hline Arecaceae & Calamus sp. L. & Pelege (Ment) & 0.57 & 2.0 \\
\hline Arecaceae & Calamus caesius Blume & Mandorou' (Ment) & 0.40 & 2.7 \\
\hline Arecaceae & Calamus sp. L & Sasa (Ment) & 0.36 & 2.6 \\
\hline Gleicheniaceae & Gleicheniaceae C.Presl & Osap (Ment) & 0.32 & 2.3 \\
\hline Arecaceae & Cocos nucifera L. & Toitet (Ment) / Kelapa (BI) & 0.19 & 3.3 \\
\hline
\end{tabular}




\section{Key informant perspectives of Mentawai medicinal and useful plants}

A total of 147 ethnospecies were cited during key informant interviews: 77 medicinal plants, and 70 plants for food, building, tool-making, firewood and ceremonial purposes. Of these, 20 plants had not been previously cited in the community survey. When shown the community freelisting activity results, all key informants agreed that the results and ranking adequately reflected the salient plants used for medicines and other purposes. Key informants made additional comments suggesting that although the plants cited during the community freelisting activity were important, factors such as ease of harvest, relative abundance, and presence in bebet uma (plants growing next to the uma) may contribute to higher rankings of some plants. Other observations suggest that some plants may have received lower rankings since survey activities took place outside flowering, fruiting and/or harvesting seasons.

\section{Significance and importance of medicinal plants and their uses}

Using the top-ranking results from the community freelisting activity, coupled with recommendations suggested in key informant interviews, 31 ethnospecies were identified as important medicinal plants for healing both physical and spiritual diseases and illnesses (Figure 5; Table 2). Of these, 35 percent were herbaceous, 19 percent trees, 16 percent shrubs, 16 percent grasses, 6 percent climbers, 3 percent palms, and 3 percent ferns. Zingiberaceae was the most represented plant family (10 species). There were minor differences between the rankings of plants cited during the community freelisting activity and those cited during the key informant interviews. Aileleppet (Graptophyllum pictum) was ranked most highly in RFC, CSI, and IV (Table 3).

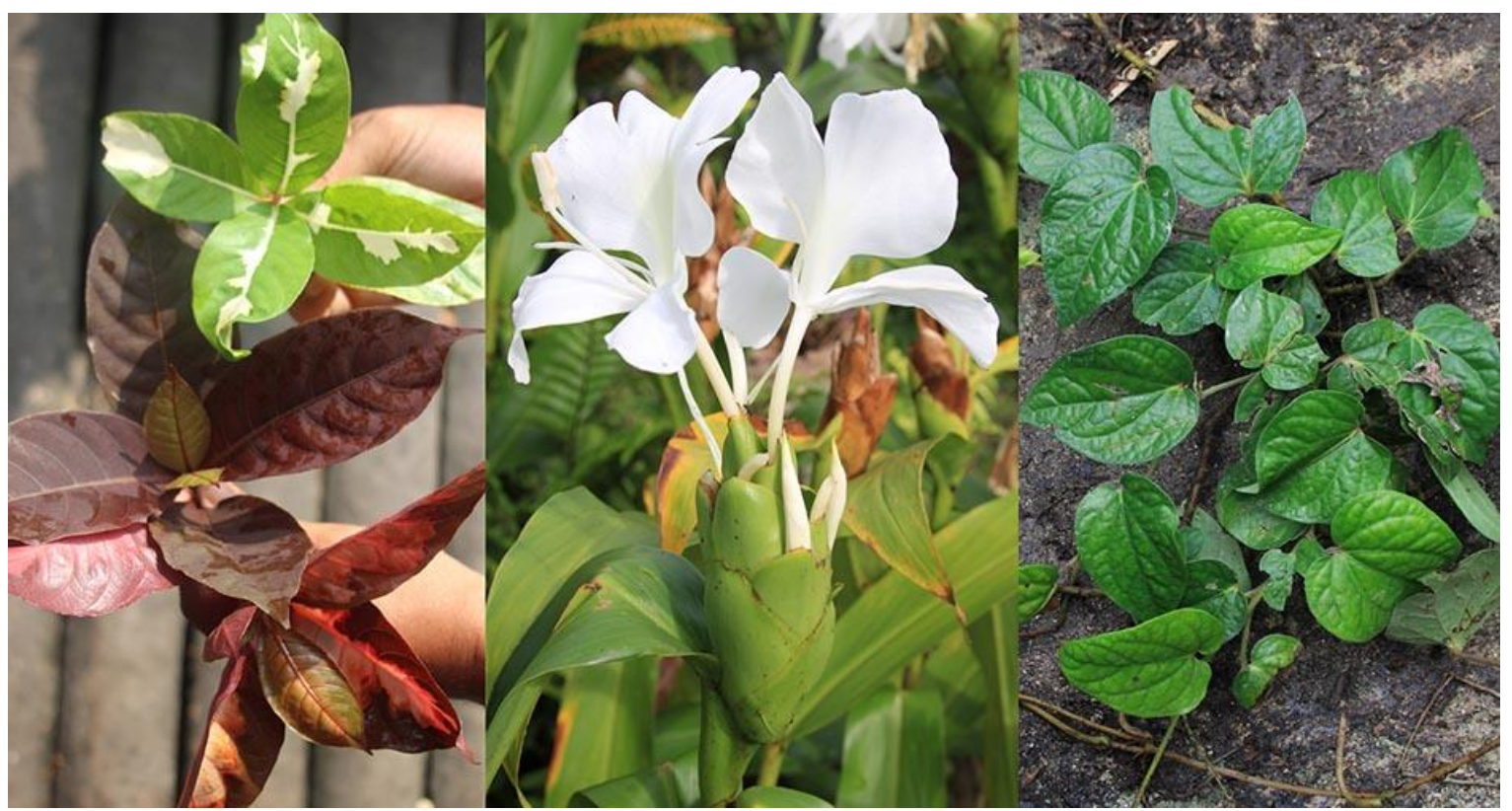

Figure 5: Three of the most frequently cited plants used for medicinal purposes by the Mentawai people living in the Sabirut and Sarereiket regions of Siberut, Mentawai Islands, Indonesia. From left to right: Aileleppet (Graptophyllum pictum (L.) Griff. (Acanthaceae)), kakainau' (Sabirut dialect) / simakainau' (Rereiket dialect) (Hedychium coronarium J.Koenig (Zingiberaceae)), and talingengeng (Piper betle L. (Piperaceae)). All three plants are readily found in bebet uma (plants in the gardens surrounding the uma). Aileleppet and kakainau' are also considered important for Mentawai ceremonies and rituals. 
Table 2. Thirty-one important medicinal plant species and examples of their uses for the Mentawai people living in the Sabirut and Sarereiket regions of Siberut, Mentawai Islands, Indonesia (M medicine, C - ceremonial, F - food, W - firewood, B - building, T - tool making / handicrafts; Ment -Mentawai language, Sib -Siberut dialect, Sab -Sabirut dialect, Sar -Rereiket dialect, BI - Bahasa Indonesia; $\mathrm{Ba}$ - bark, $\mathrm{Fl}$ - flower, $\mathrm{Fr}$ - fruit, L - leaf, $\mathrm{P}$ - pith, $\mathrm{Rh}$ - rhizome, $\mathrm{R}$ - roots, $\mathrm{S}$ - shoots, $\mathrm{St}$ - stem, $\mathrm{Ti}$ - timber, Tu - tuber / corm).

\begin{tabular}{|c|c|c|c|c|c|c|c|}
\hline $\begin{array}{l}\text { Communit } \\
y \\
\text { freelisting } \\
\text { rank }\end{array}$ & Category & Family & Scientific name & Vernacular name(s) & Plant habit & $\begin{array}{l}\text { Part } \\
\text { used }\end{array}$ & Reported uses \\
\hline 1 & MC & Acanthaceae & $\begin{array}{l}\text { Graptophyllum pictum } \\
\text { (L.) Griff. }\end{array}$ & Aileleppet (Ment) & Shrub & $L$ & $\begin{array}{l}\text { Medicine used for fevers, swelling, headaches and sore back. Can be } \\
\text { grated and used topically, or drunk as a tea. Also has significant } \\
\text { ceremonial importance, and usually features in all ceremonies. Often } \\
\text { planted at the front of a new uma. Maileppet means 'cooled', } \\
\text { indicating its use for treating heat conditions. }\end{array}$ \\
\hline 2 & MC & Zingiberaceae & $\begin{array}{l}\text { Hedychium coronarium } \\
\text { J.Koenig / Hedychium } \\
\text { flavescens Carey ex } \\
\text { Roscoe }\end{array}$ & $\begin{array}{c}\text { Kakainau' (Sab) / Simakainau' } \\
\text { (Sar) }\end{array}$ & Herbaceous & $\begin{array}{l}\mathrm{Fl}, \mathrm{Fr}, \mathrm{L}, \\
\mathrm{R}, \mathrm{S}\end{array}$ & $\begin{array}{l}\text { Used in many sikerei medicines, particularly in kisei preparations (a } \\
\text { type of spiritual illness or disease manifested in the physical body in } \\
\text { varying ways). Roots used in laggek pakop sagu. Young shoots used to } \\
\text { treat ulcers. Rotting fruits can be used to treat vomiting. The flower is } \\
\text { often worn by sikerei when walking in the forest in case medicine is } \\
\text { needed whilst away from the plant source. The leaves are worn as a skirt } \\
\text { by sikerei during ceremonial rituals. }\end{array}$ \\
\hline 3 & $\bar{M}$ & Piperaceae & Piper betle L. & $\begin{array}{c}\text { Talingengeng (Ment) / Sirih } \\
\text { (BI) }\end{array}$ & Climber & $\mathrm{L}, \mathrm{R}$ & $\begin{array}{c}\text { Used in many medicines, particularly for kisei medicines, Used in } \\
\text { laggek sasaggoi and laggek lango' preparations. Used topically to } \\
\text { treat machete wounds, or other open skin abrasions. }\end{array}$ \\
\hline 4 & MC & Zingiberaceae & Kaempferia galangal L. & $\begin{array}{l}\text { Kopuk (Sab) / Sikopuk (Sar) / } \\
\text { Kencur (BI) }\end{array}$ & Herbaceous & $\mathrm{L}, \mathrm{Rh}$ & $\begin{array}{l}\text { Used in laggek lango' and laggek leitik preparations. When delirious } \\
\text { or possessed, the smell of the leaf is used to "surprise" patients back to } \\
\text { consciousness. Rhizome can be used to treat stomach ache and fevers. }\end{array}$ \\
\hline 5 & MC & Rubiaceae & Mussaenda frondosa $\mathrm{L}$. & Mumunen (Sib) & Shrub / tree & $\begin{array}{l}\mathrm{Ba}, \mathrm{Fl}, \mathrm{L}, \\
\mathrm{R}\end{array}$ & $\begin{array}{l}\text { Used in laggek siripusou and laggek leitik remedies. A very important } \\
\text { plant used for nearly all ceremonies. The leaves are worn to signify a } \\
\text { woman is pregnant. Mumunen means buluk raumun purimanua ("leaf } \\
\text { that brings blessings for a full and happy life"). }\end{array}$ \\
\hline 6 & MFC & Zingiberaceae & Curcuma longa $\mathrm{L}$. & Kiniu (Ment) / Kunyit (BI) & Herbaceous & L, Rh & $\begin{array}{l}\text { Used as a base for many medicines. Used in food preparations. Grated } \\
\text { and used topically for treating skin infections, e.g. boils. The root is } \\
\text { grated and painted over the body and face by sikerei during } \\
\text { ceremonies. }\end{array}$ \\
\hline 7 & MC & Asparagaceae & $\begin{array}{l}\text { Cordyline fruticosa (L.) } \\
\text { A.Chev. }\end{array}$ & Boblo (Ment) & Shrub & St & $\begin{array}{l}\text { Used in medicinal preparations to treat stomach ache, mixed with a } \\
\text { variety of plant stems. Also planted at gravesites. Leaves are important } \\
\text { for ceremonies. }\end{array}$ \\
\hline 8 & $\bar{M}$ & Poaceae & $\begin{array}{l}\text { Eleusine indica (L.) } \\
\text { Gaertn. }\end{array}$ & $\begin{array}{c}\text { Ngiti-ngitit (Sib) / } \\
\text { Komensakkoko' (Sab) }\end{array}$ & Grass & $\mathrm{L}$ & Used in laggek pakop sagu preparations. \\
\hline 9 & MF & Zingiberaceae & Hornstedtia conica Ridl. & Kukuet (Ment) & Herbaceous & $\begin{array}{l}\mathrm{Fl}, \mathrm{Fr}, \mathrm{L}, \\
\mathrm{Rh}, \mathrm{St}\end{array}$ & $\begin{array}{l}\text { Often used in masisingin (sweet smelling) medicines. Used in some } \\
\text { snakebite remedies. Leaves are crushed and used whilst bathing to } \\
\text { clean the skin. Flowers are used in laggek lango' preparations. Flowers } \\
\text { seeped in hot water and drunk as a tonic. Fruits are eaten for food. }\end{array}$ \\
\hline
\end{tabular}




\begin{tabular}{|c|c|c|c|c|c|c|c|}
\hline $\begin{array}{l}\text { Communit } \\
\text { y } \\
\text { freelisting } \\
\text { rank }\end{array}$ & Category & Family & Scientific name & Vernacular name(s) & Plant habit & $\begin{array}{l}\text { Part } \\
\text { used }\end{array}$ & Reported uses \\
\hline 10 & MFC & Zingiberaceae & $\begin{array}{l}\text { Zingiber officinale } \\
\text { Roscoe }\end{array}$ & Laiga' (Ment) / Jahe (BI) & Herbaceous & $\mathrm{Rh}$ & Used to treat stomach complaints. Also valued in cooking. \\
\hline 11 & M & Poaceae & Rottboellia sp. Naezén & Boku-boku' (Ment) & Grass & $\mathrm{L}$ & $\begin{array}{c}\text { Used in laggek pakop sagu preparations. Often mixed with several } \\
\text { other leaves and roots. }\end{array}$ \\
\hline 12 & MC & Malvaceae & Hibiscus rosa-sinensis $\mathrm{L}$. & $\begin{array}{l}\text { Bekeu (Ment) / Kembang } \\
\text { sepatu (BI) }\end{array}$ & Shrub & $\mathrm{L}, \mathrm{Fl}$ & $\begin{array}{l}\text { Leaves are used in medicine to treat "inside body heat" (a type of fever). } \\
\text { Rotting flowers are also used for some medicines. Important ceremonial } \\
\text { flower worn in hair, alongside simakainau' (Hedychium coronarium / H. } \\
\text { flavescens). }\end{array}$ \\
\hline 13 & MBC T & Euphorbiaceae & $\begin{array}{l}\text { Codiaeum variegatum } \\
\text { (L.) Rumph. ex A.Juss. }\end{array}$ & Sura' (Ment) & Shrub / tree & L, St & $\begin{array}{l}\text { Used in laggek siritettet. Used to treat fungal infections and skin } \\
\text { ulcers. Placed inside the roof of the uma during ceremony. Worn on the } \\
\text { upper arms of sikerei during ceremony. Planted at the base of toitet, } \\
\text { doriat (Durio sp.) or sagu (Metroxylon sagu) tree to signify the death of } \\
\text { a clan member. The phrase masura' bagatta is inspired by this plant, } \\
\text { and translates as "there are colourful leaves growing inside us" - or, } \\
\text { thank you. }\end{array}$ \\
\hline 14 & MC & Zingiberaceae & $\begin{array}{l}\text { Alpinia galanga (L.) } \\
\text { Willd. }\end{array}$ & $\begin{array}{l}\text { Oroket (Sab) / Palakkokoai } \\
\text { (Sar) }\end{array}$ & Herbaceous & $\mathrm{Fl}, \mathrm{Fr}, \mathrm{L}, \mathrm{S}$ & $\begin{array}{c}\text { Used in many kisei medicines. Used in laggek siripusou sikatait, } \\
\text { laggek pakop sagu and laggek lango' preparations. Can be used for } \\
\text { treating headache and stomach ache. Leaves are sometimes held whilst } \\
\text { performing ceremonial dances. }\end{array}$ \\
\hline 15 & MC & Marattiaceae & $\begin{array}{l}\text { Angiopteris evecta } \\
\text { (G.Forst.) Hoffm. }\end{array}$ & Bakkat laggai (Ment) & Fern & $\mathrm{R}, \mathrm{Tu}$ & $\begin{array}{l}\text { Used in laggek pakop sagu and laggek siripusou preparations. Roots } \\
\text { are grated and covered over a sacrificial animal to protect its spirit. In } \\
\text { this ritual, sikerei ask for the animal's blessing and ask that its spirit } \\
\text { would bring more of its kind to the clan group. If the animal is hunted } \\
\text { from the forest, sikerei seek blessing from the spirit of the forest. }\end{array}$ \\
\hline 16 & $M$ & Zingiberaceae & $\begin{array}{c}\text { Etlingera coccinea } \\
\text { (Blume) S.Sakai \& } \\
\text { Nagam. }\end{array}$ & Pelekak (Sib) & Herbaceous & $\mathrm{Fl}, \mathrm{L}, \mathrm{S}, \mathrm{St}$ & $\begin{array}{c}\text { If after being sick for a long time and the body is still weak, the flower is } \\
\text { mixed with several other leaves and flowers to help restore the body's } \\
\text { energy. Kinoso (young newly blossomed flower) pelekak used in } \\
\text { laggek lango' preparations. Was also observed being used in treating } \\
\text { more complicated illnesses. }\end{array}$ \\
\hline 17 & $M$ & INDENT & INDENT & Obboi (Sib) & Grass & $\mathrm{L}, \mathrm{R}$ & $\begin{array}{c}\text { Used to treat a sweaty, hot fever. Used in laggek dugui preparations. } \\
\text { Can be used for treating stomach aches and coughs. }\end{array}$ \\
\hline 18 & MCF & Zingiberaceae & $\begin{array}{l}\text { Etlingera elatior (Jack) } \\
\text { R.M. Sm. }\end{array}$ & $\begin{array}{l}\text { Tottot mamai (Sab) / Gojo } \\
\text { (Sar) }\end{array}$ & Herbaceous & $\mathrm{Fl}, \mathrm{Fr}, \mathrm{L}, \mathrm{S}$ & $\begin{array}{l}\text { Tairatti (young flowers) are used in many medicine preparations. Gojo } \\
\text { (stems / shoots) are used to treat stomach ache. If a child hasn't taken } \\
\text { its mothers milk for several days and still refuses the breast, the leaves } \\
\text { are mixed and drunk to encourage her to return (laggek sika to'to'). } \\
\text { Used in laggek lango' preparations. Totonan (fruits) eaten and tairatti } \\
\text { often cooked with vegetables or meat. }\end{array}$ \\
\hline 19 & MFW & Myrtaceae & Psidium guajava $\mathrm{L}$. & $\begin{array}{l}\text { Jabbui (Ment) / Sabbui (Sar) } \\
\text { Jambu biji (BI) }\end{array}$ & Tree & $\mathrm{Fr}, \mathrm{L}$ & $\begin{array}{l}\begin{array}{l}\text { Leaf mixed with other leaves and drunk as a tea to treat diarrhoea. Fruit } \\
\text { eaten. }\end{array}\end{array}$ \\
\hline 20 & MC & Piperaceae & $\begin{array}{l}\text { Piper sarmentosum } \\
\text { Roxb. }\end{array}$ & Baba (Sib) & Climber & $\mathrm{L}$ & $\begin{array}{c}\text { Used in laggek lango' and laggek siripusou preparations. Can be used } \\
\text { to treat sprained or injured joints. }\end{array}$ \\
\hline
\end{tabular}




\section{Ethnobotany Research and Applications}

\begin{tabular}{|c|c|c|c|c|c|c|c|}
\hline $\begin{array}{l}\text { Communit } \\
\text { y } \\
\text { freelisting } \\
\text { rank }\end{array}$ & Category & Family & Scientific name & Vernacular name(s) & Plant habit & $\begin{array}{l}\text { Part } \\
\text { used }\end{array}$ & Reported uses \\
\hline 21 & M & Acoraceae & Acorus calamus $\mathrm{L}$. & $\begin{array}{l}\text { Butek-butek (Sab) / Sumamra } \\
\text { (Sar) }\end{array}$ & Grass & $\mathrm{L}$ & $\begin{array}{c}\text { Used in laggek lango' sikebukat preparations. Often mixed with a } \\
\text { variety of Zingiberaceae species and covered over the forehead and } \\
\text { back of neck. }\end{array}$ \\
\hline 22 & M B CW & Fabaceae & $\begin{array}{l}\begin{array}{l}\text { Erythrina subumbrans } \\
\text { (Hassk.) Merr. }\end{array} \\
\end{array}$ & Mancuat (Sab) / Matcuat (Sar) & Tree & $\mathrm{Ba}$ & $\begin{array}{c}\text { Used in many laggek lango' preparations, especially for women after } \\
\text { giving birth. Flower worn by newly initiated sikerei. }\end{array}$ \\
\hline 23 & MCF & Zingiberaceae & $\begin{array}{l}\text { Alpinia malaccensis } \\
\text { C.Presl }\end{array}$ & $\begin{array}{c}\text { Kojo koddiai (Sib) / } \\
\text { Kasisingin (Sab) / Pasisingin } \\
\text { (Sar) }\end{array}$ & Herbaceous & $\begin{array}{l}\mathrm{Fl}, \mathrm{Fr}, \mathrm{L}, \\
\mathrm{Rh}, \mathrm{St}\end{array}$ & $\begin{array}{l}\text { Used in many medicines including laggek siripusou preparations. Leaf } \\
\text { used to close obbuk (bamboo) whilst cooking. Fruit is eaten. }\end{array}$ \\
\hline 24 & $\mathrm{MC}$ & Zingiberaceae & $\begin{array}{l}\text { Curcuma zanthorrhiza } \\
\text { Roxb. }\end{array}$ & $\begin{array}{l}\text { Kiniu sailu' (Ment) / } \\
\text { Temulawak (BI) }\end{array}$ & Herbaceous & $\mathrm{Fl}, \mathrm{Rh}$ & $\begin{array}{l}\text { Used in laggek pakop sagu and laggek siripusou preparations. Used } \\
\text { to heal the spirit of a person. When a sick, the plant is grated and mixed } \\
\text { with parts of the gojo (Etlingera elatior) plant and taken to the forest to } \\
\text { entice the spirit of that person to take on the colour of its flower. }\end{array}$ \\
\hline 25 & MW & Annonaceae & $\begin{array}{l}\text { Cananga odoratum } \\
\text { (Lam.) Hook.f. \& } \\
\text { Thomson }\end{array}$ & Poak (Sib) & Tree & $\begin{array}{c}\mathrm{Ba}, \mathrm{L}, \mathrm{R}, \\
\mathrm{Ti}\end{array}$ & $\begin{array}{c}\text { Used in laggek lango', laggek sibebelak and laggek sisaksana } \\
\text { preparations. Can be used as firewood. Used in medicines when giving } \\
\text { birth. Can be used to treat sprained or injured joints. }\end{array}$ \\
\hline 29 & MC & Acanthaceae & $\begin{array}{l}\text { Justicia gendarussa } \\
\text { Burm.f. }\end{array}$ & $\begin{array}{l}\text { Pakasele (Sab) / Pangasele } \\
\text { (Sar) }\end{array}$ & Shrub & L, R, St & $\begin{array}{c}\text { Used in many kisei medicines. Used in laggek leitiki preparations. Can } \\
\text { be used to treat intestinal complaints. }\end{array}$ \\
\hline 32 & MCT & Arecaceae & $\begin{array}{l}\text { Daemonorops } \\
\text { angustifolia } \\
\text { (Griff.) Mart. }\end{array}$ & Rui (Sab) / Labi (Sar) & Palm & L, St & $\begin{array}{l}\text { Used in laggek pakop sagu preparations. Leaves are used to seal the } \\
\text { top of the roof of the uma. Part of the palm is used to make gigiok (a } \\
\text { tool used to crush and grate medicinal plants). New growth at the top } \\
\text { of the palm can be eaten. }\end{array}$ \\
\hline 38 & M & Commelinaceae & $\begin{array}{l}\text { Amischotolype } \\
\text { mollissima (Blume) } \\
\text { Hassk. }\end{array}$ & Lemu-lemu' (Ment) & Shrub & $\mathrm{Ba}, \mathrm{L}, \mathrm{St}$ & Used in laggek appu and laggek lango' preparations. \\
\hline 45 & MC & Poaceae & $\begin{array}{l}\text { Cymbopogon citratus } \\
\text { (DC.) Stapf }\end{array}$ & $\begin{array}{l}\text { Siat-siat (Sab) / Jiat-jiat (Sar) / } \\
\text { Serai (BI) }\end{array}$ & Grass & L, St & $\begin{array}{l}\begin{array}{l}\text { Used in many medicinal preparations. Stem used to add flavour to } \\
\text { foods. }\end{array} \\
\end{array}$ \\
\hline 53 & M & Onagraceae & Ludwigia linifolia Poir. & $\begin{array}{l}\text { Lotte-lotte' (Sab) / Mumru } \\
\text { tuktuk (Sar) }\end{array}$ & Herbaceous & $L, R$ & $\begin{array}{c}\text { Used in laggek simatiet, laggek sasaggoi, laggek baga siripusou } \\
\text { and laggek pakop sagu preparations. Called mumru tuktuk as its } \\
\text { flower form resembles tuktuk mumru (prawn whiskers). }\end{array}$ \\
\hline 61 & M & Annonaceae & $\begin{array}{l}\text { Goniothalamus sp. } \\
\text { (Blume) Hook.f \& } \\
\text { Thomson }\end{array}$ & Simuinek (Sar) & Tree & L, St & $\begin{array}{c}\text { Used in laggek pakop sagu, laggek lango', laggek besit ute' and } \\
\text { laggek sasaggoi preparations. }\end{array}$ \\
\hline
\end{tabular}


Table 3. Comparison between three ethnobotanical quantitative measures (RFC - Relative frequency of citation, CSI - Cultural significance index, IV - Importance value) ranking the top 31 plant species considered important medicinal resources for Mentawai communities in the Sabirut and Sarereiket regions of Siberut, Mentawai Islands, Indonesia. Plants have been ordered according to RFC during key informant interviews. (Ment -Mentawai language, Sib -Siberut dialect, Sab - Sabirut dialect, Sar -Rereiket dialect, BI - Bahasa Indonesia).

\begin{tabular}{|c|c|c|c|c|c|c|}
\hline \multirow{2}{*}{ Family } & \multirow{2}{*}{ Scientific name } & \multirow{2}{*}{ Vernacular name(s) } & \multirow{2}{*}{$\begin{array}{c}\begin{array}{c}\text { Comm- } \\
\text { unity }\end{array} \\
\text { RFC }\end{array}$} & \multicolumn{3}{|c|}{ Key informant } \\
\hline & & & & RFC & CSI & IV \\
\hline Acanthaceae & $\begin{array}{l}\text { Graptophyllum } \\
\text { pictum (L.) Griff. }\end{array}$ & Aileleppet (Ment) & 0.44 & 0.67 & 4.00 & 0.48 \\
\hline Zingiberaceae & $\begin{array}{l}\text { Hedychium } \\
\text { coronarium J.Koenig / } \\
\text { Hedychium flavescens } \\
\text { Carey ex Roscoe }\end{array}$ & $\begin{array}{l}\text { Kakainau' (Sab) / } \\
\text { Simakainau' (Sar) }\end{array}$ & 0.43 & 0.57 & 3.14 & 0.10 \\
\hline Rubiaceae & $\begin{array}{l}\text { Mussaenda frondosa } \\
\text { L. }\end{array}$ & Mumunen (Sib) & 0.22 & 0.52 & 1.71 & 0.05 \\
\hline Zingiberaceae & Kaempferia galanga L. & $\begin{array}{l}\text { Kopuk (Sab) / Sikopuk (Sar) / } \\
\text { Kencur (BI) }\end{array}$ & 0.32 & 0.43 & 1.93 & 0.05 \\
\hline Poaceae & $\begin{array}{l}\text { Eleusine indica (L.) } \\
\text { Gaertn. }\end{array}$ & $\begin{array}{l}\text { Ngiti-ngitit (Sib) / } \\
\text { Komensakkoko' (Sab) }\end{array}$ & 0.14 & 0.43 & 1.93 & - \\
\hline Zingiberaceae & $\begin{array}{l}\text { Etlingera coccinea } \\
\text { (Blume) S.Sakai \& } \\
\text { Nagam. }\end{array}$ & Pelekak (Sib) & 0.07 & 0.33 & 0.75 & 0.05 \\
\hline Piperaceae & Piper betle L. & $\begin{array}{l}\text { Talingengeng (Ment) / Sirih } \\
\text { (BI) }\end{array}$ & 0.33 & 0.29 & 0.64 & 0.05 \\
\hline Asparagaceae & $\begin{array}{l}\text { Cordyline fruticosa } \\
\text { (L.) A.Chev. }\end{array}$ & Boblo (Ment) & 0.15 & 0.29 & 1.71 & - \\
\hline Poaceae & $\begin{array}{l}\text { Rottboellia sp. } \\
\text { Naezén }\end{array}$ & Boku-boku' (Ment) & 0.10 & 0.29 & 0.64 & 0.05 \\
\hline Marattiaceae & $\begin{array}{l}\text { Angiopteris evecta } \\
\text { (G.Forst.) Hoffm }\end{array}$ & Bakkat laggai (Ment) & 0.07 & 0.24 & 1.07 & - \\
\hline Fabaceae & $\begin{array}{l}\text { Erythrina subumbrans } \\
\text { (Hassk.) Merr. }\end{array}$ & $\begin{array}{l}\text { Mancuat (Sab) / Matcuat } \\
\text { (Sar) }\end{array}$ & 0.03 & 0.24 & 1.07 & - \\
\hline Commelinaceae & $\begin{array}{l}\text { Amischotolype } \\
\text { mollissima (Blume) } \\
\text { Hassk. }\end{array}$ & Lemu-lemu' (Ment) & 0.02 & 0.24 & 1.07 & - \\
\hline Zingiberaceae & Curcuma longa L. & Kiniu (Ment) / Kunyit (BI) & 0.15 & 0.19 & 1.14 & - \\
\hline Zingiberaceae & $\begin{array}{l}\text { Alpinia galanga (L.) } \\
\text { Willd. }\end{array}$ & $\begin{array}{l}\text { Oroket (Sab) / Palakkokoai } \\
\text { (Sar) }\end{array}$ & 0.07 & 0.19 & 0.86 & 0.05 \\
\hline Arecaceae & $\begin{array}{l}\text { Daemonorops } \\
\text { angustifolia } \\
\text { (Griff.) Mart. }\end{array}$ & Rui (Sab) / Labi (Sar) & 0.03 & 0.19 & 0.43 & - \\
\hline Acanthaceae & $\begin{array}{l}\text { Justicia gendarussa } \\
\text { Burm.f. }\end{array}$ & $\begin{array}{l}\text { Pakasele (Sab) / Pangasele } \\
\text { (Sar) }\end{array}$ & 0.03 & 0.19 & 0.86 & - \\
\hline Zingiberaceae & $\begin{array}{l}\text { Hornstedtia conica } \\
\text { Ridl. }\end{array}$ & Kukuet (Ment) & 0.12 & 0.14 & 0.64 & - \\
\hline Zingiberaceae & $\begin{array}{l}\text { Zingiber officinale } \\
\text { Roscoe }\end{array}$ & Laiga' (Ment) / Jahe (BI) & 0.11 & 0.14 & 0.64 & - \\
\hline Malvaceae & $\begin{array}{l}\text { Hibiscus rosa-sinensis } \\
\mathrm{L} \text {. }\end{array}$ & $\begin{array}{l}\text { Bekeu (Ment) / Kembang } \\
\text { sepatu (BI) }\end{array}$ & 0.09 & 0.14 & 0.86 & - \\
\hline INDENT & INDENT & Obboi (Sib) & 0.06 & 0.14 & 0.64 & - \\
\hline Piperaceae & $\begin{array}{l}\text { Piper sarmentosum } \\
\text { Roxb. }\end{array}$ & Baba (Sib) & 0.05 & 0.14 & 0.64 & - \\
\hline Zingiberaceae & $\begin{array}{l}\text { Etlingera elatior (Jack) } \\
\text { R.M. Sm. }\end{array}$ & $\begin{array}{l}\text { Tottot mamai (Sab) / Gojo } \\
\text { (Sar) }\end{array}$ & 0.04 & 0.14 & 0.32 & - \\
\hline Poaceae & $\begin{array}{l}\text { Cymbopogon citratus } \\
\text { (DC.) Stapf }\end{array}$ & $\begin{array}{l}\text { Siat-siat (Sab) / Jiat-jiat (Sar) } \\
\text { / Serai (BI) }\end{array}$ & 0.02 & 0.14 & 0.64 & - \\
\hline Onagraceae & Ludwigia linifolia Poir. & $\begin{array}{l}\text { Lotte-lotte' (Sab) / Mumru } \\
\text { tuktuk (Sar) }\end{array}$ & 0.01 & 0.14 & 0.32 & - \\
\hline Annonaceae & $\begin{array}{l}\text { Goniothalamus sp. } \\
\text { (Blume) Hook.f. \& } \\
\text { Thomson }\end{array}$ & Simuinek (Sar) & 0.01 & 0.14 & 0.32 & - \\
\hline Euphorbiaceae & $\begin{array}{l}\text { Codiaeum variegatum } \\
\text { (L.) Rumph. ex A.Juss. }\end{array}$ & Sura' (Ment) & 0.08 & 0.10 & 0.57 & - \\
\hline Myrtaceae & Psidium guajava L. & $\begin{array}{l}\text { Jabbui (Ment) / Jambu biji } \\
\text { (BI) }\end{array}$ & 0.05 & 0.10 & 0.43 & - \\
\hline
\end{tabular}




\begin{tabular}{|c|c|c|c|c|c|c|}
\hline \multirow{2}{*}{ Family } & \multirow{2}{*}{ Scientific name } & \multirow{2}{*}{ Vernacular name(s) } & \multirow{2}{*}{$\begin{array}{c}\begin{array}{c}\text { Comm- } \\
\text { unity }\end{array} \\
\text { RFC }\end{array}$} & \multicolumn{3}{|c|}{ Key informant } \\
\hline & & & & RFC & CSI & IV \\
\hline Zingiberaceae & $\begin{array}{l}\text { Alpinia malaccensis } \\
\text { (Burm.f.) Roscoe }\end{array}$ & $\begin{array}{l}\text { Kojo koddiai (Sib) / } \\
\text { Kasisingin (Sab) / Pasisingin } \\
\text { (Sar) }\end{array}$ & 0.04 & 0.10 & 0.21 & - \\
\hline Annonaceae & $\begin{array}{l}\text { Cananga odoratum } \\
\text { (Lam.) Hook.f. \& } \\
\text { Thomson }\end{array}$ & Poak (Sib) & 0.03 & 0.10 & 0.21 & - \\
\hline Acoraceae & Acorus calamus L. & $\begin{array}{l}\text { Butek-butek (Sab) / } \\
\text { Sumamra (Sar) }\end{array}$ & 0.05 & 0.05 & 0.21 & - \\
\hline Zingiberaceae & $\begin{array}{l}\text { Curcuma zanthorrhiza } \\
\text { Roxb. }\end{array}$ & $\begin{array}{l}\text { Kiniu sailu' (Ment) / } \\
\text { Temulawak (BI) }\end{array}$ & 0.04 & 0.05 & 0.21 & - \\
\hline
\end{tabular}

\section{Significance and importance of other useful plants}

Using the top-ranking citations from the freelisting activity, along with recommendations provided in key informant interviews, 32 ethnospecies were identified as the most useful plants across five categories (nutritional, ceremonial, building, tool-making and firewood) (Figure 6; Table 4). Of these, 63 percent were trees, 9 percent palms, 9 percent rattan kinds, 9 percent herbaceous, 3 percent grasses, 3 percent ferns and 3 percent shrubs. Arecaceae was the most represented botanical family (six species identified). Sagu (Metroxylon sagu) ranked highest for RFC, CSI and IV (Table 5). Plants that were considered more valuable for medicinal use and previously cited in Table 2 were excluded from Table 4. Despite sagu being listed as the most important food source for the Mentawai, it was observed that most families living in resettlement communities were more likely to eat rice, rather than sagu at every meal. Further investigation as to the reasons why this is the case have yet to be examined, but it is assumed that convenience and a lack of access to clan lands and resources has influenced this choice.

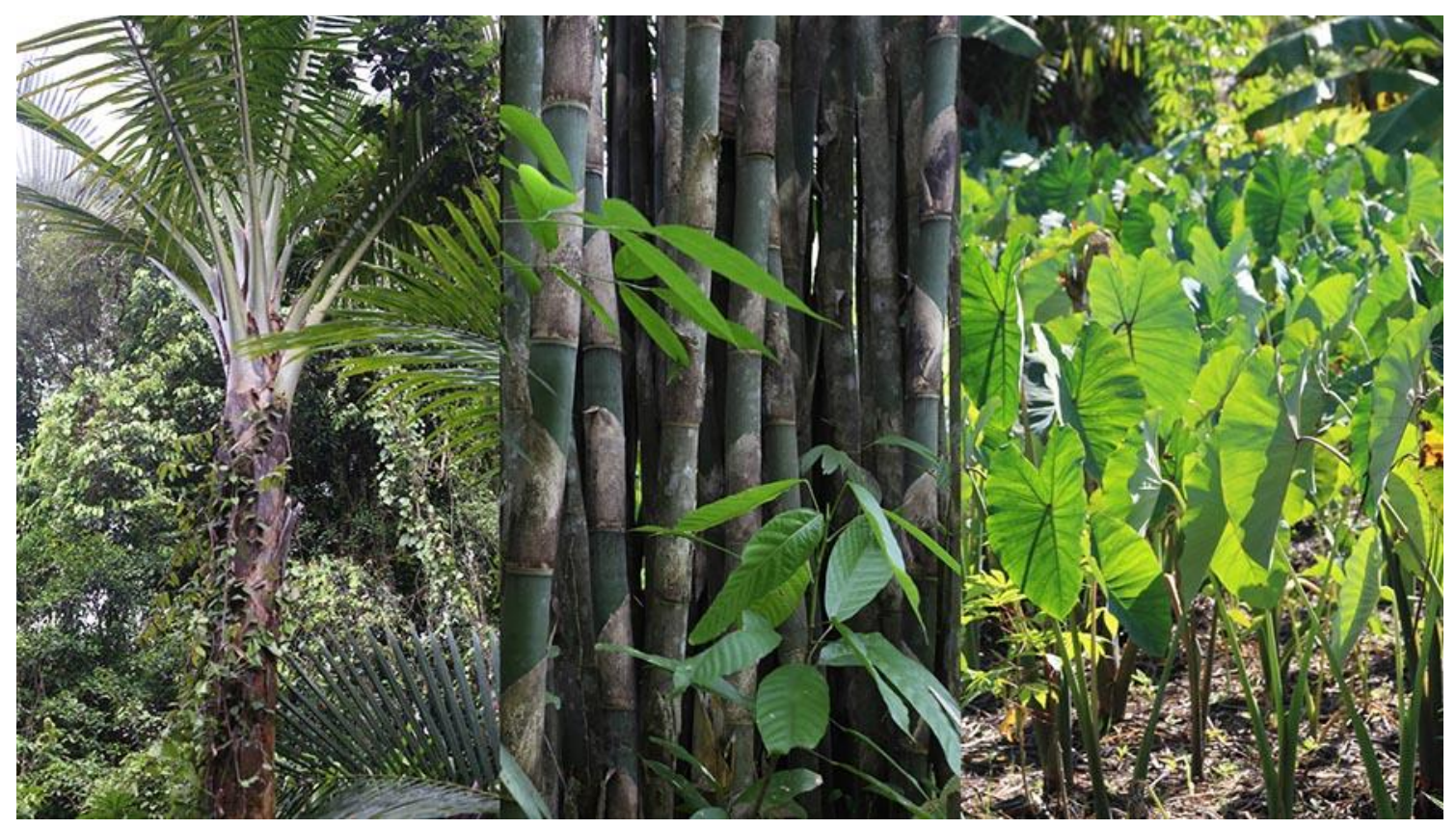

Figure 6. Three of the most frequently cited plants considered useful for food, building, firewood, ceremony, and/or tool-making purposes by the Mentawai living in the Sabirut and Sarereiket regions of Siberut, Mentawai Islands. From left to right: Sagai (Sabirut dialect) / Sagu (Rereiket dialect) (Metroxylon sagu Rottb. (Arecaceae)), manggeak and obbuk (bamboo types), and gette' (Colocasia esculenta (L.) Schott (Araceae)). 
Table 4. Thirty-two important plant resources and examples of their uses for the Mentawai people living in the Sabirut and Sarereiket regions of Siberut, Mentawai Islands, Indonesia (M - medicine, C - ceremonial, F - food, W - firewood, B - building, T - tool making / handicrafts; Ment - Mentawai language, Sib -Siberut dialect, Sab -Sabirut dialect, Sar -Rereiket dialect, BI - Bahasa Indonesia; Ba - bark, Fl - flower, $\mathrm{Fr}$ - fruit, L - leaf, $\mathrm{P}$ - pith, Rh - rhizome, R - roots, S - shoots, St - stem, Ti - timber, Tu - tuber / corm).

\begin{tabular}{|c|c|c|c|c|c|c|c|}
\hline $\begin{array}{l}\text { Commun } \\
\text { ity } \\
\text { freelistin } \\
\text { g rank }\end{array}$ & Category & Family & Scientific name & Vernacular name(s) & $\begin{array}{l}\text { Plant } \\
\text { habit }\end{array}$ & $\begin{array}{l}\text { Part } \\
\text { used }\end{array}$ & Reported uses \\
\hline 1 & $\begin{array}{l}\text { BCFM T } \\
\text { W }\end{array}$ & Arecaceae & $\begin{array}{l}\text { Metroxylon sagu } \\
\text { Rottb. }\end{array}$ & Sagai (Sab) / Sagu (Sar) & Palm & $\begin{array}{l}\mathrm{Ba}, \mathrm{L}, \mathrm{P}, \\
\mathrm{R}, \mathrm{Sh}\end{array}$ & $\begin{array}{l}\text { Pith is a staple food source for humans, and all } \\
\text { domesticated animals. Leaves are used for roofing, } \\
\text { cooking sagu, cooking utensils (e.g. bibibip), tappiri } \\
\text { (basket for storing processed sagu). Spine of leaf is as } \\
\text { duck food, and for making babaklu kerei (sacred sikerei } \\
\text { box) and bolobop (large carrying basket). The skin is } \\
\text { used dried for firewood (salot), and as a long-lasting } \\
\text { torch. Skin is also used for making opa and karagjug } \\
\text { (carrying baskets). Roots and shoots used in medicines. } \\
\text { Skin and leaves used as building materials. Fronds are } \\
\text { used to burn off feathers and hair of ceremonial meats. } \\
\text { An important plant traded in alat toga negotiations } \\
\text { (where possessions of wealth are negotiated as a bride } \\
\text { dowry). }\end{array}$ \\
\hline 2 & $\begin{array}{l}\text { BCFM T } \\
\text { W }\end{array}$ & Poaceae & Bamboo types & $\begin{array}{l}\text { Manggeak (Ment) / } \\
\text { Obbuk (Ment) / Bambu } \\
\text { (BI) }\end{array}$ & Grass & Sh & $\begin{array}{l}\text { Used for obbuk - used like an oven for cooking meats, } \\
\text { vegetables, sagu, and to store and carry food and water. } \\
\text { Important firewood source. Young shoots are eaten and } \\
\text { used in medicine preparations. Used for many building } \\
\text { purposes - e.g. small structures / shelter, animal pens, } \\
\text { fencing, flooring, tool-making, roofing (kalasau - long } \\
\text { pieces used to hold sagu leaves). Used to build goro } \\
\text { jobatoinan (aqueduct). Ceremonial significance - e.g. } \\
\text { uma juju simagre (home of the spirits). Used to store } \\
\text { medicines (uma laggek). Worn by women whilst fishing } \\
\text { to store fish (lagasat). Leaves are used as flooring for } \\
\text { roiget gou'-gou' (chicken) basket). }\end{array}$ \\
\hline 3 & CF M T & Araceae & $\begin{array}{l}\text { Colocasia esculenta } \\
\text { (L.) Schott }\end{array}$ & $\begin{array}{l}\text { Gette' (Ment) / Keladi } \\
\text { (Bl) }\end{array}$ & $\begin{array}{l}\text { Herbaceou } \\
\mathrm{s}\end{array}$ & L, St, Tu & $\begin{array}{l}\text { Staple food source for humans. Tubers, stems and young } \\
\text { leaves are eaten. Leaves can be used as a tobacco } \\
\text { substitute. Older, yellowing leaves are used in several } \\
\text { medicines. Important plant traded in alat toga } \\
\text { negotiations. Important ceremonial food. }\end{array}$ \\
\hline 4 & $\begin{array}{l}\text { B C F M T } \\
\text { W }\end{array}$ & Arecaceae & Cocos nucifera L. & $\begin{array}{l}\text { Toitet (Ment) / Kelapa } \\
\text { (BI) }\end{array}$ & Palm & $\mathrm{Fr}, \mathrm{L}$ & $\begin{array}{l}\text { Staple food source for humans and domesticated } \\
\text { animals. Young coconut water is drunk and used in }\end{array}$ \\
\hline
\end{tabular}




\begin{tabular}{|c|c|c|c|c|c|c|c|}
\hline $\begin{array}{l}\text { Commun } \\
\text { ity } \\
\text { freelistin } \\
\text { g rank }\end{array}$ & Category & Family & Scientific name & Vernacular name(s) & $\begin{array}{l}\text { Plant } \\
\text { habit }\end{array}$ & $\begin{array}{l}\text { Part } \\
\text { used }\end{array}$ & Reported uses \\
\hline & & & & & & & $\begin{array}{l}\text { medicinal preparations. Husk is burnt to smoke out } \\
\text { insects, and braided to make rope. Oil used in cooking } \\
\text { and medicines. Bole used for construction purposes. Shell } \\
\text { used to make a variety of tools including sisip } \\
\text { (ceremonial soup spoon) and baklu (box used to store } \\
\text { sacred sikerei paraphernalia). Leaves are used in cooking. } \\
\text { Leaves are woven to carry gou'-gou' between owners } \\
\text { (tapir). Important palm traded in alat toga negotiations. }\end{array}$ \\
\hline 5 & CFMT & Musaceae & Musa spp. L. & $\begin{array}{l}\text { Bago' (Sab) / Mago' } \\
\text { (Sar) / Pisang (BI) }\end{array}$ & $\begin{array}{l}\text { Herbaceou } \\
\mathrm{s}\end{array}$ & $\mathrm{Fl}, \mathrm{Fr}, \mathrm{L}$ & $\begin{array}{l}\text { Staple food source for humans, saina' (pigs) and gou'- } \\
\text { gou'. Leaves are traditionally worn as a skirt by women } \\
\text { whilst fishing. Flowers used in medicines. Leaves are dried } \\
\text { and used as cigarette rolling papers. Leaves used to wrap } \\
\text { and protect food. }\end{array}$ \\
\hline 6 & B T & Arecaceae & Calamus sp. L. & Pelege (Ment) & Rotan & L, S, St & $\begin{array}{l}\text { Used to make karagjug (large woven basket carried on } \\
\text { the back), opa (small woven basket carried on the back), } \\
\text { orek (basket to store cooked sagu), letcu (bracelet), } \\
\text { roiget (gou'-gou' basket), talatat (storage basket). } \\
\text { Leaves used medicinally to treat intestinal worms. Used as } \\
\text { rope. Shoots used in laggek pakop sagu. }\end{array}$ \\
\hline 7 & B M T W & Dipterocarpaceae & $\begin{array}{l}\text { Shorea pauciflora } \\
\text { King }\end{array}$ & Katuka (Ment) & Tree & $\mathrm{Ba}, \mathrm{Ti}$ & $\begin{array}{l}\text { Important structural timber. Used to carve abak (dug-out } \\
\text { canoe). Bark used in medicinal preparations. Large boles } \\
\text { can be sold from IDR } 2,000,000 \text {. }\end{array}$ \\
\hline 8 & B F W & Malvaceae & Durio spp. Adans. & Doriat (Ment) & Tree & $\mathrm{Fr}, \mathrm{R}, \mathrm{Ti}$ & $\begin{array}{l}\text { Seasonal food source for humans, and domesticated and } \\
\text { wild animals. Large roots are used to carve tegge } \\
\text { (machete) handles. Seeds are replanted. Used for } \\
\text { construction. Important tree traded in alat toga } \\
\text { negotiations. }\end{array}$ \\
\hline 9 & B T & Arecaceae & $\begin{array}{l}\text { Calamus caesius } \\
\text { Blume }\end{array}$ & Mandorou' (Ment) & Rotan & St & $\begin{array}{l}\text { Used to make karagjug, opa, roiget and other small } \\
\text { baskets (e.g. tuku). Used to restring skulls and hung to } \\
\text { protect the uma. Used as rope for a variety of purposes. }\end{array}$ \\
\hline 10 & B T & Arecaceae & $\begin{array}{l}\text { Daemonorops sp. } \\
\text { Blume }\end{array}$ & Sasa (Ment) & Rotan & St & $\begin{array}{l}\text { Used to make bola (floor mats), garangat (small axe). } \\
\text { Used to tie bibibilit gajuma (drum skins), tingan jajat } \\
\text { (cultural paraphernalia hung in the uma). If someone } \\
\text { steals something, but denies, the two parties agree to cut } \\
\text { a piece of sasa. If the accused person was lying, within a } \\
\text { week they would become sick, and die. Can be sold as } \\
\text { income. }\end{array}$ \\
\hline
\end{tabular}




\begin{tabular}{|c|c|c|c|c|c|c|c|}
\hline $\begin{array}{l}\text { Commun } \\
\text { ity } \\
\text { freelistin } \\
\text { g rank }\end{array}$ & Category & Family & Scientific name & Vernacular name(s) & $\begin{array}{l}\text { Plant } \\
\text { habit }\end{array}$ & $\begin{array}{l}\text { Part } \\
\text { used }\end{array}$ & Reported uses \\
\hline 11 & $\mathrm{CMT}$ & Gleicheniaceae & $\begin{array}{l}\text { Gleicheniaceae } \\
\text { C.Presl }\end{array}$ & Osap (Ment) & Fern & L, St & $\begin{array}{l}\text { Used to make letcu, letcu baklu (ties for sikerei } \\
\text { keepsake box). Leaves used in medicinal preparations, } \\
\text { particularly in some laggek kisei variations. Leaves used } \\
\text { in healing ceremonies. }\end{array}$ \\
\hline 12 & BCTW & Apocynaceae & $\begin{array}{l}\text { Alstonia spatulata } \\
\text { Blume }\end{array}$ & $\begin{array}{l}\text { Kakatdut (Sab) / Gite } \\
\text { (Sar) / Pulai (BI) }\end{array}$ & Tree & $\mathrm{Ti}$ & $\begin{array}{l}\text { Used for carving tools and important cultural artefacts, } \\
\text { including jaraik (sacred symbol to prevent harmful } \\
\text { influences entering the uma). Cabinetry timber. Used to } \\
\text { make floorboards. Previously used to make coffins. }\end{array}$ \\
\hline 13 & B T W & Dipterocarpaceae & $\begin{array}{l}\text { Hopea } \\
\text { dryobalanoides Miq. }\end{array}$ & Mancemi (Ment) & Tree & $\mathrm{Ti}$ & $\begin{array}{l}\text { Important structural timber - used for beams, joists, } \\
\text { rafters, floorboards. Used to carve paluga (oar). Used to } \\
\text { carve decorative pieces for the uma. }\end{array}$ \\
\hline 14 & B T W & Rhizophoraceae & $\begin{array}{l}\text { Rhizophora apiculata } \\
\text { Blume }\end{array}$ & Bakat (Ment) / Bakau (BI) & Tree & $\mathrm{Ti}$ & $\begin{array}{l}\text { Used for firewood. Important building resource, } \\
\text { particularly around the coastal areas where tidal } \\
\text { inundation may occur. }\end{array}$ \\
\hline 15 & B C M T & Dipterocarpaceae & $\begin{array}{l}\text { Dipterocarpus } \\
\text { fusiformis P.S.Ashton }\end{array}$ & $\begin{array}{l}\text { Elagat (Sab) / Kara-kara } \\
\text { (Sar) / Kruing (BI) }\end{array}$ & Tree & $\begin{array}{l}\text { Ba, Ti, } \\
\text { Resin }\end{array}$ & $\begin{array}{l}\text { Important structural timber - used to create footbridges, } \\
\text { floorboards, verandas. Rotting wood is host to an edible } \\
\text { worm. Before kerosene became accessible, oil from the } \\
\text { wood was used to light lanterns. Large boles can be sold } \\
\text { for IDR 2-3,000,000. Used in kisei medicines. }\end{array}$ \\
\hline 16 & $\mathrm{CT}$ & Arecaceae & $\begin{array}{l}\text { Arenga pinnata } \\
\text { (Wurmb) Merr. }\end{array}$ & $\begin{array}{l}\text { Poula (Ment) / Katsaila } \\
\text { (Ment) / Anau (BI) / Aren } \\
\text { (BI) }\end{array}$ & Palm & $\begin{array}{l}\mathrm{Fl}, \mathrm{L}, \mathrm{St}, \\
\mathrm{Ti}\end{array}$ & $\begin{array}{l}\text { Leaves are arranged in the hair of sikerei during } \\
\text { ceremony. At the beginning of a ceremony, a small piece } \\
\text { is tied on the ngalou (necklace) of all those present to } \\
\text { signify blessing and protection during the ceremony. } \\
\text { Leaves are used to carry saina' and monkeys between } \\
\text { locations. Leaves also used to make brooms. Trunk used } \\
\text { to make gajuma (drum), sasaala (hammer used to make } \\
\text { kabit (loincloth)). The flower collects water, which is } \\
\text { drunk. Hairs are used as a strainer in processing sagu. }\end{array}$ \\
\hline 17 & B T W & Myristicaceae & $\begin{array}{l}\text { Horsfieldia sp. Blume } \\
\text { ex DC. }\end{array}$ & Ribbu (Ment) & Tree & $\mathrm{Ti}$ & $\begin{array}{l}\text { Highly valued structural timber. Used for dumundai } \\
\text { (structural stumps for uma). Source of firewood. }\end{array}$ \\
\hline 18 & B W & Euphorbiaceae & $\begin{array}{l}\text { Macaranga sp. } \\
\text { Thouars }\end{array}$ & Katumanggei (Ment) & Tree & $\mathrm{Ti}$ & $\begin{array}{l}\text { Timber used for beams in the uma. Used to create } \\
\text { overhead storage in the uma. Used for firewood. }\end{array}$ \\
\hline 19 & F M W & Melastomataceae & $\begin{array}{l}\text { Melastoma } \\
\text { sylvaticum Blume }\end{array}$ & $\begin{array}{l}\text { Sibetu (Sab) / } \\
\text { Eru'teinung (Sar) }\end{array}$ & Tree & $\mathrm{L}, \mathrm{Ti}$ & $\begin{array}{l}\text { Used for firewood. Leaves are used as gou'-gou' bedding } \\
\text { in roiget. Leaves used to treat stomach ache. Fruit is } \\
\text { eaten and used in medicinal preparations. Worms found } \\
\text { in bole are used as fishing bait. Worms can also be eaten. }\end{array}$ \\
\hline 20 & F M & Convolvulaceae & Ipomoea sp. L. & $\begin{array}{l}\text { Gobi' (Sab) / Siputeteket } \\
\text { (Sar) / Ubi jalar (BI) }\end{array}$ & $\begin{array}{l}\text { Herbaceou } \\
\mathrm{s}\end{array}$ & Tu & Corm eaten. Leaves can be eaten, although rare. \\
\hline
\end{tabular}




\begin{tabular}{|c|c|c|c|c|c|c|c|}
\hline $\begin{array}{l}\text { Commun } \\
\text { ity } \\
\text { freelistin } \\
\text { g rank }\end{array}$ & Category & Family & Scientific name & Vernacular name(s) & $\begin{array}{l}\text { Plant } \\
\text { habit }\end{array}$ & $\begin{array}{l}\text { Part } \\
\text { used }\end{array}$ & Reported uses \\
\hline 21 & B W & Malvaceae & $\begin{array}{l}\text { Pentace triptera } \\
\text { Mast. }\end{array}$ & Kaboi (Sib) & Tree & $\mathrm{Ba}, \mathrm{Ti}$ & $\begin{array}{l}\text { Important structural timber for building uma. Can be } \\
\text { used to carve abak. Bark used to make dedebu (bucket } \\
\text { used for processing sagu). Large boles can be sold from } \\
\text { IDR } 1,800,000 \text {. Can be used as firewood. }\end{array}$ \\
\hline 22 & B C T W & Dipterocarpaceae & $\begin{array}{l}\text { Shorea } s p . \text { Roxb. ex } \\
\text { C.F.Gaertn. }\end{array}$ & Karai (Ment) & Tree & $\mathrm{Ti}$ & $\begin{array}{l}\text { Structural timber used for walls, doors and floorboards. } \\
\text { Wood is used to carve jaraik (sacred carving). Used to } \\
\text { make karaibo (shield). Used to carve abak and paluga. }\end{array}$ \\
\hline 23 & B F W & Sapindaceae & $\begin{array}{l}\text { Nephelium } \\
\text { lappaceum L. }\end{array}$ & $\begin{array}{l}\text { Bairabbit (Sib) / } \\
\text { Rambutan (BI) }\end{array}$ & Tree & $\mathrm{Fr}, \mathrm{Ti}$ & Seasonal food source. Source of firewood. \\
\hline 24 & FM & Euphorbiaceae & Manihot sp. Mill. & $\begin{array}{l}\text { Bairatti (Sab) / Sipukaju } \\
\text { (Sar) / Ubi kayu (BI) }\end{array}$ & Shrub & $\mathrm{Tu}$ & Important food source. \\
\hline 25 & F M W & Meliaceae & $\begin{array}{l}\text { Lansium parasiticum } \\
\text { (Osbeck) K.C.Sahni \& } \\
\text { Bennet }\end{array}$ & $\begin{array}{l}\text { Selak (Sab) / Tiat (Sar) / } \\
\text { Siamung (Sar) / Duku } \\
\text { (BI) / Langsat (BI) }\end{array}$ & Tree & $\mathrm{Fr}, \mathrm{R}$ & Seasonal food source. Used in laggek leitik preparations. \\
\hline 26 & B W & Euphorbiaceae & $\begin{array}{l}\text { Macaranga tanarius } \\
\text { (L.) Müll.Arg. }\end{array}$ & $\begin{array}{l}\text { Pari-pari (Sab) / Ottot } \\
\text { (Sar) }\end{array}$ & Tree & $\mathrm{L}, \mathrm{Ti}$ & $\begin{array}{l}\text { Timber used to carve tegge and other small knife } \\
\text { handles. Used in construction. Leaves are used for } \\
\text { wrapping and carrying medicines. }\end{array}$ \\
\hline 39 & B W & Myristicaceae & $\begin{array}{l}\text { Horsfieldia irya } \\
\text { (Gaertn.) Warb. }\end{array}$ & Roat (Sab) / Doat (Sar) & Tree & $\mathrm{L}, \mathrm{Ti}$ & $\begin{array}{l}\text { Used as firewood. Leaves can be seeped in water to add a } \\
\text { masisingin (sweet) flavour. }\end{array}$ \\
\hline 40 & B W & INDENT & INDENT & Manggugu' (Ment) & Tree & $\mathrm{R}, \mathrm{Ti}$ & $\begin{array}{l}\text { Used as firewood. Used as flooring timber for uma. Roots } \\
\text { are used in medicinal preparations. }\end{array}$ \\
\hline 54 & B T W & INDENT & INDENT & Bolasi (Ment) & Tree & $\mathrm{Ti}$ & $\begin{array}{l}\text { Long burning firewood. Timber is used for building and } \\
\text { for handicrafts. }\end{array}$ \\
\hline 56 & F M W & Sapindaceae & $\begin{array}{l}\text { Nephelium } \\
\text { cuspidatum Blume }\end{array}$ & $\begin{array}{l}\text { Kapa (Sab) / Babaet } \\
\text { (Sar) }\end{array}$ & Tree & $\mathrm{Fr}, \mathrm{L}, \mathrm{Ti}$ & $\begin{array}{l}\text { Used as firewood. Used to carve tuddukat. Leaves and } \\
\text { bark used in medicines. Fruit is eaten. Fruit can be used to } \\
\text { dye clothes. Used in laggek lango' preparations. }\end{array}$ \\
\hline 58 & W & Myristicaceae & $\begin{array}{l}\text { Gymnacranthera sp. } \\
\text { (A.DC.) Warb. }\end{array}$ & Ngalou pataddekat (Sar) & Tree & $\mathrm{Ti}$ & Source of firewood. \\
\hline 67 & B W & Apocynaceae & $\begin{array}{l}\text { Kibatalia arborea } \\
\text { (Blume) G.Don }\end{array}$ & Toilat (Ment) & Tree & $\mathrm{Ti}$ & $\begin{array}{l}\text { Used for firewood. Used for building, particularly for } \\
\text { window frames. }\end{array}$ \\
\hline
\end{tabular}


Table 5. Comparison between three ethnobotanical quantitative measures (RFC - Relative frequency of citation, CSI - Cultural significance index, IV - Importance value) ranking the top 32 plant species considered important food, building, tool-making, firewood and ceremonial resources for the Mentawai communities in the Sabirut and Sarereiket regions of Siberut, Mentawai Islands, Indonesia. Plants have been ordered according to RFC during key informant interviews. (Ment - Mentawai language, Sib -Siberut dialect, Sab -Sabirut dialect, Sar - Rereiket dialect, BI - Bahasa Indonesia).

\begin{tabular}{|c|c|c|c|c|c|c|}
\hline \multirow[t]{2}{*}{ Family } & \multirow{2}{*}{ Scientific name } & \multirow[t]{2}{*}{ Vernacular name(s) } & \multirow{2}{*}{$\begin{array}{c}\text { Comm- } \\
\text { unity } \\
\text { RFC }\end{array}$} & \multicolumn{3}{|c|}{ Key informant } \\
\hline & & & & RFC & CSI & IV \\
\hline Arecaceae & Metroxylon sagu Rottb. & Sagai (Sab) / Sagu (Sar) & 0.34 & 0.90 & 8.00 & 0.95 \\
\hline Araceae & $\begin{array}{l}\text { Colocasia esculenta (L.) } \\
\text { Schott }\end{array}$ & Gette' (Ment) / Keladi (BI) & 0.17 & 0.71 & 6.32 & 0.10 \\
\hline Arecaceae & Calamus sp. L. & Pelege (Ment) & 0.10 & 0.57 & 1.89 & 0.33 \\
\hline Dipterocarpaceae & Shorea pauciflora King & Katuka (Ment) & 0.09 & 0.57 & 1.89 & 0.52 \\
\hline Myristicaceae & $\begin{array}{l}\text { Horsfieldia sp. Blume ex } \\
\text { DC. }\end{array}$ & Ribbu (Ment) & 0.04 & 0.52 & 1.16 & 0.29 \\
\hline Poaceae & Various & $\begin{array}{l}\text { Manggeak (Ment) / } \\
\text { Obbuk (Ment) / Bambu } \\
\text { (BI) }\end{array}$ & 0.18 & 0.43 & 3.79 & 0.29 \\
\hline Arecaceae & $\begin{array}{l}\text { Arenga pinnata } \\
\text { (Wurmb) Merr. }\end{array}$ & $\begin{array}{l}\text { Poula (Ment) / Katsaila } \\
\text { (Ment) / Anau (BI) / Aren } \\
(\mathrm{BI})\end{array}$ & 0.04 & 0.33 & 1.47 & 0.19 \\
\hline Arecaceae & Calamus caesius Blume & Mandorou' (Ment) & 0.07 & 0.29 & 0.95 & 0.24 \\
\hline Dipterocarpaceae & $\begin{array}{l}\text { Hopea dryobalanoides } \\
\text { Miq. }\end{array}$ & Mancemi (Ment) & 0.05 & 0.29 & 0.63 & 0.05 \\
\hline Arecaceae & Cocos nucifera L. & Toitet (Ment) / Kelapa (BI) & 0.15 & 0.24 & 1.68 & 0.05 \\
\hline INDENT & INDENT & Bolasi (Ment) & 0.02 & 0.24 & 0.79 & 0.19 \\
\hline Musaceae & Musa spp. L. & $\begin{array}{l}\text { Bago' (Sab) / Mago' (Sar) } \\
\text { / Pisang (BI) }\end{array}$ & 0.13 & 0.19 & 0.84 & \\
\hline Apocynaceae & $\begin{array}{l}\text { Kibatalia arborea } \\
\text { (Blume) G.Don }\end{array}$ & Toilat (Ment) & 0.01 & 0.19 & 0.63 & 0.10 \\
\hline Arecaceae & $\begin{array}{l}\text { Daemonorops sp. } \\
\text { Blume }\end{array}$ & Sasa (Ment) & 0.06 & 0.14 & 0.47 & 0.05 \\
\hline Gleicheniaceae & Gleicheniaceae C.Presl & Osap (Ment) & 0.05 & 0.14 & 0.47 & 0.05 \\
\hline Apocynaceae & $\begin{array}{l}\text { Alstonia spatulata } \\
\text { Blume }\end{array}$ & $\begin{array}{l}\text { Kakatdut (Sab) / Gite } \\
\text { (Sar) / Pulai (BI) }\end{array}$ & 0.05 & 0.14 & 0.32 & 0.05 \\
\hline Melastomataceae & $\begin{array}{l}\text { Melastoma sylvaticum } \\
\text { Blume }\end{array}$ & $\begin{array}{l}\text { Sibetu (Sab) / Eru'teinung } \\
\text { (Sar) }\end{array}$ & 0.03 & 0.14 & 0.11 & \\
\hline Stemonuraceae & $\begin{array}{l}\text { Stemonurus } \\
\text { malaccensis (Mast.) } \\
\text { Sleumer }\end{array}$ & Manggugu' (Ment) & 0.03 & 0.14 & 0.47 & 0.10 \\
\hline Sapindaceae & $\begin{array}{l}\text { Nephelium cuspidatum } \\
\text { Blume }\end{array}$ & Kapa (Sab) / Babaet (Sar) & 0.01 & 0.14 & 0.95 & 0.05 \\
\hline Myristicaceae & $\begin{array}{l}\text { Gymnacranthera (A.DC.) } \\
\text { Warb. }\end{array}$ & Ngalou pataddekat (Sar) & 0.01 & 0.14 & 0.47 & 0.05 \\
\hline Dipterocarpaceae & $\begin{array}{l}\text { Dipterocarpus } \\
\text { fusiformis P.S.Ashton }\end{array}$ & $\begin{array}{l}\text { Elagat (Sab) / Kara-kara } \\
\text { (Sar) / Kruing (BI) }\end{array}$ & 0.04 & 0.10 & 0.11 & \\
\hline Malvaceae & Pentace triptera Mast. & Kaboi (Sib) & 0.03 & 0.10 & 0.21 & 0.05 \\
\hline Dipterocarpaceae & $\begin{array}{l}\text { Shorea } s p . \text { Roxb. ex } \\
\text { C.F.Gaertn. }\end{array}$ & Karai (Ment) & 0.03 & 0.10 & 0.21 & 0.05 \\
\hline Myristicaceae & $\begin{array}{l}\text { Horsfieldia irya } \\
\text { (Gaertn.) Warb. }\end{array}$ & Roat (Sab) / Doat (Sar) & 0.03 & 0.10 & 0.21 & 0.05 \\
\hline Malvaceae & Durio spp. Adans. & Doriat (Ment) & 0.07 & 0.05 & 0.16 & \\
\hline Rhizophoraceae & $\begin{array}{l}\text { Rhizophora apiculata } \\
\text { Blume }\end{array}$ & Bakat (Ment) / Bakau (BI) & 0.05 & 0.05 & 0.11 & \\
\hline Euphorbiaceae & Macaranga sp. Thouars & Katumanggei (Ment) & 0.04 & 0.05 & 0.11 & \\
\hline
\end{tabular}




\begin{tabular}{lllllc}
\hline \multirow{2}{*}{ Family } & Scientific name & Vernacular name(s) & $\begin{array}{c}\text { Comm- } \\
\text { unity }\end{array}$ & \multicolumn{2}{c}{ Key informant } \\
\cline { 3 - 6 } Euphorbiaceae & $\begin{array}{l}\text { Macaranga tanarius (L.) } \\
\text { Müll.Arg. }\end{array}$ & $\begin{array}{l}\text { Pari-pari (Sab) / Ottot } \\
(\text { Sar) }\end{array}$ & 0.03 & 0.05 & 0.08 \\
\hline Euphorbiaceae & Manihot sp. Mill. & $\begin{array}{l}\text { Bairatti (Sab) / Sipukaju } \\
\text { (Sar) / Ubi kayu (BI) }\end{array}$ & 0.03 & 0.00 & 0.21 \\
\hline Convolvulaceae & Ipomoea sp. L. & $\begin{array}{l}\text { Gobi' (Sab) / Siputeteket } \\
\text { (Sar) / Ubi jalar (BI) }\end{array}$ & 0.03 & Not cited & 0.21 \\
\hline Sapindaceae & $\begin{array}{l}\text { Nephelium lappaceum } \\
\text { L. }\end{array}$ & $\begin{array}{l}\text { Bairabbit (Sib) / } \\
\text { Rambutan (BI) }\end{array}$ & 0.03 & Not cited & 0.21 \\
\hline Meliaceae & $\begin{array}{l}\text { Lansium parasiticum } \\
\text { (Osbeck) K.C.Sahni \& } \\
\text { Bennet }\end{array}$ & $\begin{array}{l}\text { Selak (Sab) / Tiat (Sar) / } \\
\text { Siamung (Sar) / Duku (BI) } \\
\text { / Langsat (BI) }\end{array}$ & 0.05 \\
\hline
\end{tabular}

\section{Community perceptions of Mentawai traditional ecological knowledge}

Community surveying sought to reveal Mentawai attitudes towards their TEK and whether the community held any concerns about 'why plant knowledge may be eroding or lost'(Figure 7). Nearly all survey respondents agreed that Mentawai plant knowledge is important for the future of Mentawai, particularly as food and medicine resources (98 percent; 95\% confidence interval (Cl) 97\%-99\%). One respondent stated:

Our plant knowledge is very important. Our plants have many benefits, especially in treating sickness. In our traditions, we understand that our plants are the source of our life. We cannot navigate our way in the world without them.

Mentawai woman, Muntei, February 2019.

When asked what would happen if this knowledge is lost, many believed life would become difficult (38 percent; 95\% Cl 34\%-43\%) or become poor (18 percent; 95\% Cl 15\%-21\%). Conversely, 19 percent of respondents believed nothing would happen if plant knowledge were lost (95\% Cl 16\%-22\%).

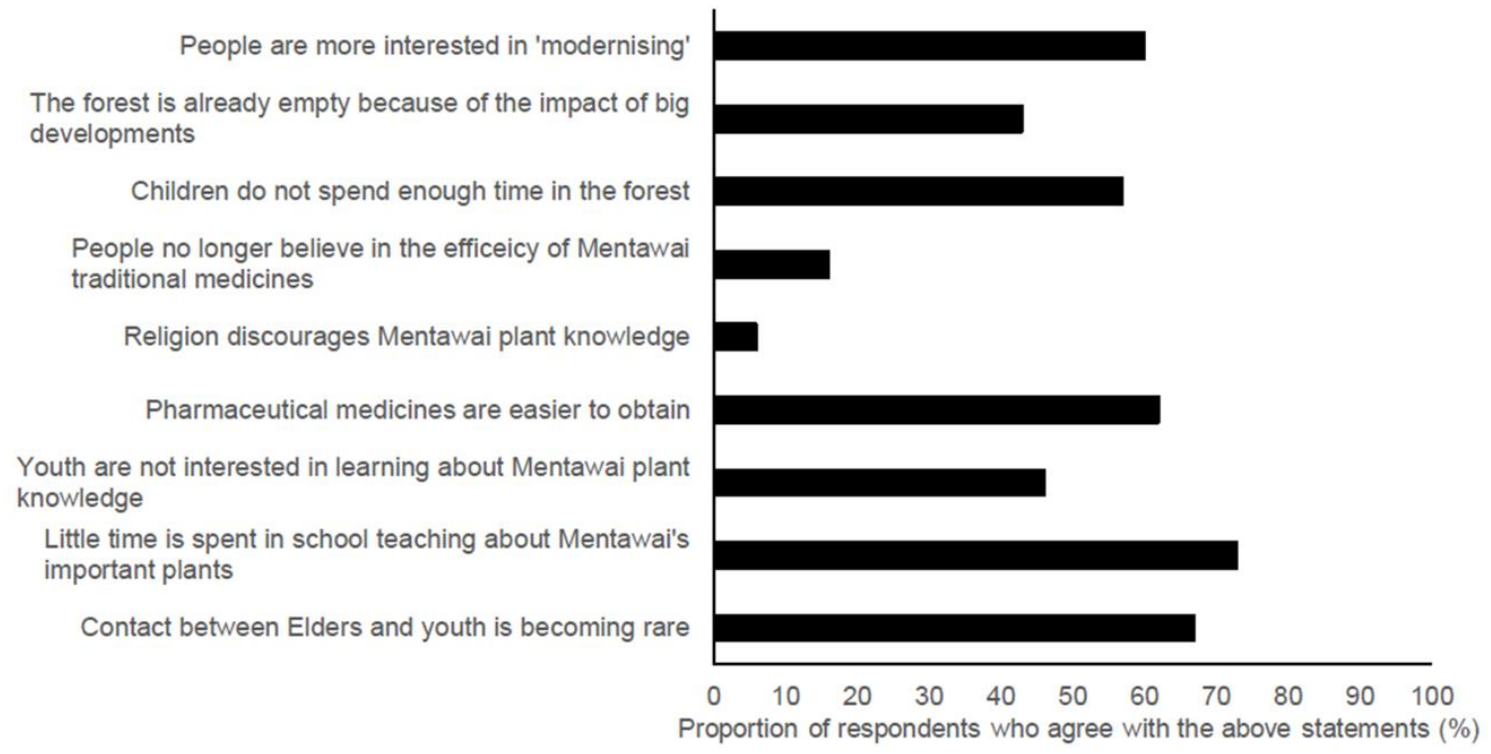

Figure 7. A sample of community perceptions for why Mentawai traditional ecological knowledge might be eroding in the Sabirut and Sarereiket regions of Siberut, Mentawai Islands, Indonesia $(n=494)$. Most respondents felt the time spent between sikebbu'kat (respected elders) and youth is becoming rare due to changes in family social patterns, and because many children now attend formal schools, where little time is spent teaching students about Mentawai's important plants and ecosystem functions.

Half of all respondents felt people were too busy working other jobs to practice Mentawai TEK (95\% Cl 44\%-53\%), and over half felt that people were more interested in 'modernising' (95\% Cl 56\%-64\%). Two-thirds of respondents 
believed TEK is eroding because contact between youth and sikebbu'kat is decreasing (95\% Cl 63\%-71\%). Half of all respondents felt that children and youth do not seem interested in learning about Mentawai plants $(95 \% \mathrm{Cl}$ 41\%-50\%), nor spend enough time in the forest (95\% Cl 53\%-62\%). Additionally, 73 percent of respondents felt too little time was spent in school learning about traditional plant uses (95\% Cl $70 \%-77 \%)$. Eighty percent of respondents believed the loss of Mentawai TEK may become exasperated due to the immigration of young people from Mentawai to other parts of Indonesia or abroad (95\% Cl 47\%-56\%).

Half of all respondents believed Siberut's forests still contain important plants that can be utilised on a day-to-day basis (95\% Cl 45\%-53\%). Seventy-four percent of respondents believed the forest would never become 'empty', because Mentawai plant knowledge and the principles and practices of arat sabulungan ensure the forest is protected, cared for, and important species are replanted (95\% Cl 70\%-78\%). However, a third of respondents identified a correlation between the loss of Mentawai knowledge and the depletion of forest resources $(95 \% \mathrm{Cl}$ $32 \%-41 \%)$. The community perceived that the greatest threats to Siberut's forest resources were deforestation caused by large-scale developments, and lack of protective measures or support from the government. A large portion felt that the forest had already been damaged due to extensive development, including unsuitable logging operations or land conversion (43 percent; $95 \% \mathrm{Cl} 39 \%-47 \%$ ). The majority did not believe that religion discouraged traditional practices (88 percent; $95 \% \mathrm{Cl} 86 \%-91 \%$ ).

\section{Transmission of Mentawai traditional ecological knowledge}

Two-thirds of respondents felt they knew enough about Mentawai plant knowledge (95\% Cl 62\%-70\%). This was reflected in the perception that respondents felt they had learnt enough primarily through daily activities from a young age (22 percent; $95 \% \mathrm{Cl} 18 \%-25 \%$ ), or had been encouraged by other community members to learn about Mentawai plants (16 percent; $95 \% \mathrm{Cl} 13 \%-20 \%$ ) (Figure 8). However, of those unsure or felt they did not know enough about Mentawai TEK, most felt it was because they had never been taught (30 percent; $95 \% \mathrm{Cl} 26 \%-33 \%$ ).

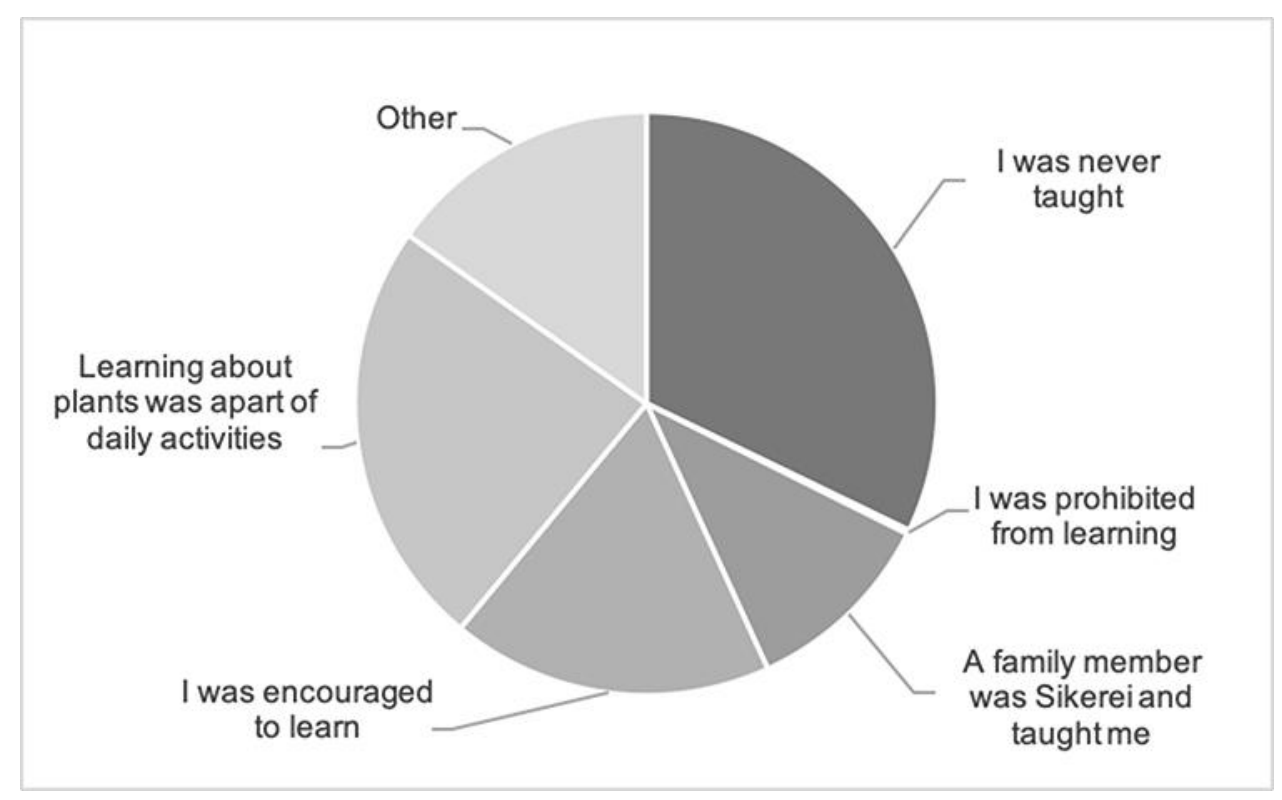

Figure 8. Reasons why the Sabirut and Sarereiket communities of Siberut, Mentawai Islands, Indonesia, feel they have or have not an adequate level of Mentawai plant knowledge. A third of respondents felt they knew very little about Mentawai plant knowledge because they did not have the opportunity to learn. Those who did feel as though they knew enough recognised that most learnings had taken place during daily household activities.

Respondents generally perceived that all community members were responsible for teaching and sharing Mentawai plant knowledge. However, many respondents recognised the responsibility of the sikerei in safeguarding traditional plant knowledge (23 percent; 95\% Cl 20\%-27\%). Half of all respondents believed that the knowledge had not been forgotten by sikerei or sikebbu'kat ( $95 \% \mathrm{Cl} 47 \%-56 \%$ ). There were mixed responses as to whether 
ways of teaching traditional plant use had changed, and whether sikerei and sikebbu'kat spent enough time in the forest practicing traditional knowledge.

Ninety percent of key informants reported having taught, or passed on, their plant knowledge $(95 \% \mathrm{Cl} 78 \%-100 \%)$, explaining that Mentawai ecological knowledge is usually taught 'hands-on' as the opportunity or need is presented. Many respondents added they had only taught others if they had been directly asked by those interested in learning. Nearly all respondents raised concerns about the lack of knowledge or interest by the younger generation, with several lamenting that 'young people are ignorant of the benefits of Mentawai's plants'. One respondent worried that if they were not asked to teach Mentawai TEK, then

"...our knowledge will become extinct. Our people will no longer remember who we are, or how to survive."

Mentawai man, Puro, April 2019.

Despite concerns raised by sikebbu'kat and sikerei about threats to the transmission of Mentawai TEK, nearly all respondents from the community survey agreed that Mentawai plant knowledge remains essential for their future, stating:

"The life of the Mentawai cannot be separated from the forest and its plants. Mentawai plants are the breath of everything."

Sikebbu'kat, Muntei, February 2019.

Despite the results suggesting young (12-25 years of age) respondents felt they knew enough about Mentawai plants and TEK (see Figure 8), comments made by key informants, coupled with observations made during the study period, suggest otherwise. For children and youth living in resettlement communities, it was observed that considerably less time is spent in the forest where plant knowledge is shared amongst peers and siblings. While children living in uma communities spend most of the day exploring the forest or carrying out home duties utilizing forest resources surrounding the uma. In this way, it was observed that children from a young age understand Mentawai's forests' ecological and cultural functions. These interactions were rarely observed in resettlement communities, which may be attributed to new socio-economic conditions driving the loss of traditional knowledge - similar to those drivers discussed in Reyes-Garcia et al. (2013).

Other observations made during the study period infer that fewer young people are on the path to becoming sikerei. This observation may be cause for concern, given the extensive knowledge held by the sikerei and the integral role they play in maintaining the health and wellbeing of the Mentawai people. It was also observed in the study period that there can be significant variation between each sikerei and their knowledge and practice of medicinal plants, each having their own unique methods. Though the community collectively knows many plants and their uses, the variation between sikerei and knowledge holders was seen to be an important component of Siberut's cultural and biological diversity.

\section{Observations on the use of medicinal plants in the community}

Observations during the research period established an emerging trend: that is, that resettled communities are more likely to choose pharmaceutical medicines, only returning to traditional medicines and healing systems as a last resort. This is supported by the suggestion in the community survey that over half of respondents felt that people were more interested in modernizing than practicing TEK (see Figure 7). Discussions with Dr S. Tahmasebian from the Madobag government health centre (Puskesmas) revealed similar concerns: community members no longer utilise traditional plant medicines, and instead choose to visit a Puskesmas expecting a quick fix. She stated:

"There are so many herbs and leaves in the forest that can keep the Mentawai people healthy, but they aren't using them. They don't ask the sikerei to help, but come to [the Puskesmas] expecting pharmaceutical medicines. It's not sustainable, nor is it healthy. When they were younger, they didn't suffer from many of the illnesses and diseases they experience today. Now their children are often sick, when they shouldn't be."

S. Tahmasebian, Muarasiberut, May 2019.

Changes in healthcare preference may be underlined by changing values and forms of wealth: traditionally, clan groups would have access to pigs, chickens, fruits, and other important tradable items to exchange for sikerei medicines, healing ceremonies and other associated traditions. However, it was observed during the study period that those now living within resettlement communities generally have limited access to these traditional forms of wealth, instead now relying on the national currency. Though this has benefits in being able to access pharmaceutical medicines, the cost comparison is vast. For example, during the study period it was found that a course of antibiotics at the local pharmacy, without needing to see the doctor, may cost between IDR 8,000 - 
20,000. (approximately USD 0.57 - 1.42) Comparatively, when factoring payment for services, travel costs, purchase of sacrificial animals, and provision of other gifts, foods, etc., for rituals, sikerei medicines and the associated ceremonial rituals can cost upwards of IDR 400,000 (approximately USD 28) depending on the severity of illness and required treatments. Though it was observed that community members are becoming less likely to utilise traditional healing systems, many respondents from the community survey still emphasized the importance of Mentawai's plants for treating disease, stating:

"Not all pharmacy drugs are able to heal our people. Disease cannot always be treated by medical treatment or pharmaceuticals."

Mentawai woman, Muntei, February 2019.

"Without Mentawai plant medicines and rituals, disease cannot be cured. Without the

Mentawai forest, we wouldn't have our health."

Mentawai man, Maileppet, March 2019.

Contrastingly, those respondents living in uma communities were more likely to utilise traditional medicines to treat illness or disease. Many were disinterested in using pharmaceutical medicines, and were unlikely to utilise these services unless compelled as a final option. However, it was perceived that these preferences are partly due to limited access to the national currency, and the relative isolation of uma communities from allopathic healthcare options. For example, where there was an option to receive fast-acting medicines to treat headaches, sore joints or diarrhea from visiting tourists, uma clan members would often welcome medicines given by foreigners.

\section{Observations of Mentawai ecological practices and interactions}

During the observation period, it was perceived that many Mentawai traditional farming practices align with key principles of regenerative agriculture or permaculture (Rodale Institute 2015). For example, Gou'-gou' (chickens) and ducks run freely between crops, cattle are grazed as a small herd and rotated regularly, pugette'an are established between sagu trees, and mago', toitet, bairabbit and pinang are scattered between crops or plantations (Figure 9). Additionally, it was noticed that the Mentawai do not practice slash and burn agriculture growing fields are cut, and plant matter is left to rot for several years before returning to utilise the land for crops, resulting in aerated soil high in organic matter. However, some fields near Muntei village had been burnt during the observation period before replanting gette'. When asked why this might be the case, one Mentawai man explained that when larger trees are cut down in mone their stumps are usually burned prior to replanting the field. It was speculated that fields that were burnt may have been owned by immigrants to Mentawai.

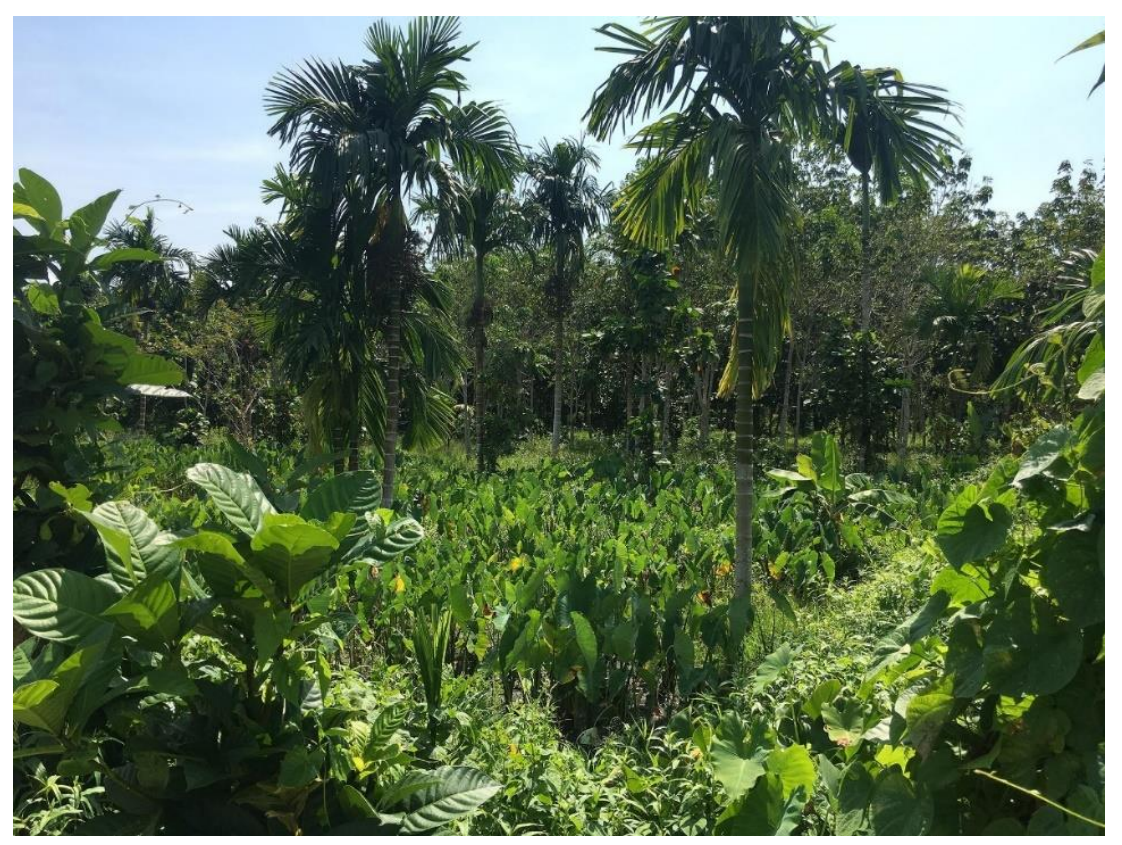

Figure 9. Pugette'an (traditional taro field) intercropped with a subsidised crop of pinang (Areca catechu L. (Arecaceae)). In the foreground are coppiced ngirip / irip (Barringtonia racemosa (L.) Spreng. (Lecythidaceae)) used to fence the field, and behind, a small plantation of rubber trees (Hevea brasiliensis (Willd. ex A.Juss.) Müll.Arg. (Euphorbiaceae)). Muntei village, Sabirut region, Siberut, Mentawai Islands, Indonesia, June 2019. 
It was also noted during the observation period that fields are often fenced using coppiced ngirip / irip (Barringtonia racemosa (L.) Spreng. (Lecythidaceae)), a practice that has been shown to enhance biodiversity across agricultural landscapes (León \& Harvey 2006). Fertiliser, herbicides and pesticides are usually not used, though, observations of farmers spraying poison to control ferns and grasses along garden paths in resettlement communities were made during the research period. It was also observed where customary land tenure is no longer observed, deforestation, sediment run-off and pollution are more apparent (though this requires further study to validate these initial observations).

\section{Authors notes on collaboration with Indigenous researchers}

Collaboration with Mentawai researchers was central to the implementation of the research activities and analysis, and is the first of its kind to emerge from Mentawai. Inclusive and transparent approaches aimed to ensure the study's objectives and methods procured accurate and meaningful outcomes, and were suitable within local contexts. When participants were informed that data gathered during the research period would be returned to the community for their own use, and to develop knowledge-sharing resources for their children, it was observed that participants were more motivated to offer in-depth and accurate responses. Engaging a team of Mentawai researchers also gave a better insight into cultural expectations and procedures, resulting in what was believed to be a more culturally appropriate approach, thus stimulating greater support and interest from sikerei, sikebbu'kat, local government and community members. Furthermore, as native speakers of the Sabirut and Rereiket dialects, it is believed that the inclusion of Mentawai peoples as researchers, not just informants, greatly reduced the probability of error in listening or spelling words and meanings in the Mentawai language. Additional perceived benefits from the project include: collaborators increased sense of ownership over and empowerment in preserving Mentawai TEK; collaborator and community increased awareness of Siberut's unique biosphere; and, increased awareness of international conservation agenda and protocol. Supporting the study's approach, one collaborating researcher commented:

I knew our forest was important, but I didn't realise just how many important plants -
particularly medicines - were in such a small part of the forest. If our people living in the
villages knew this, we wouldn't give our land away so freely. We must remember our
ancestors' knowledge. We need to keep investigating. We must share our findings with our
community.
Y. Mendrofa, YPBM Chairman and collaborating researcher, Matotonan, May 2019

\section{Discussion}

The use of medicinal plants and the impact on Siberut's biodiversity

Since tropical climates provide ideal conditions for the spread of diseases (State of the Tropics 2019) and that the Mentawai diet may be considered "calorie deficient" (Conway 1998), traditional plant medicine plays a critical role in supporting the health of the Mentawai. Access to traditional healthcare systems remains vital for both resettled and forest uma communities due to their perceived efficacy and cultural significance. If Mentawai traditional medicinal plants are no longer used or perceived as important, less value may be attributed to the forest resource, and less attention will be directed toward preserving Siberut's biome (Hamilton \& Hamilton 2006). Furthermore, as the number of youth actively pursuing the path of the sikerei declines, the diversity of Mentawai plant knowledge may also diminish. Since culture and biodiversity are "inextricably linked" (Posey 1999), changes in values, social structures, and patterns of use of Mentawai's medicinal plants may impact Siberut's biodiversity.

\section{Mentawai ecological practices and interactions supporting Siberut's biodiversity}

Mentawai's indigenous agricultural practices are small-scale, diverse, culturally relevant, flexible and adaptable characteristics essential for resilient, viable food systems (Lin et al. 2011, World Resources Institute 2019). Pumonean are highly diverse, and are important genetic resources for conserving Siberut's tree diversity (Indra et al. 2017). Moreover, pumonean traditional practices have significant cultural importance, providing crops integral to Mentawai's food culture. Gardens surrounding the uma are composed of frequently used medicinal, ceremonial, fuel and food plants based on the needs or preferences of the uma clan group. These gardens and pumonean vary in size, but are generally of high floristic diversity, require very little labour input, and contribute to the supply of important nutritional, cultural and economic plant species (Kumar \& Nair 2004). By inter-cropping a range of commercial and subsistent species, Siberut's pumonean and uma gardens provide vital food, medicine, timber and other resources for the Mentawai community - particularly for resettled communities who have limited access to more remote forest resources (Kumar \& Nair 2004, Mutua et al. 2014). 
Given the considerable impact of industrial agriculture on biodiversity (Laurance et al. 2014, Lin et al. 2011), practices like those traditionally applied by the Mentawai people contribute substantially to supporting Siberut's biodiversity (Kumar \& Nair 2004). Suppose traditional agricultural ecosystems are damaged, or traditional practices discontinued. In that case, there is a possibility that native or endemic plant species may become extinct, thereby causing a decline in the wellbeing of the Mentawai and their biome (WWF 1980). Mentawai traditional practices have the potential to safeguard local food security, maintain long-term sustainability, and regenerate humandegraded ecosystems (Garrity 2004, Kumar \& Nair 2004, McNeely \& Schroth 2006, Retnowati 2003). Furthermore, by not practicing slash and burn agricultural methods, a lack of fire across Siberut's landscapes ensures the soil is never exposed, thus preventing significant erosion, run-off and stream sedimentation - an important practice given Siberut's fragile soils originate from hardened mud sediments, and that riparian zones are subject to extreme erosion (Suhandi et al. 2002; WWF 1980).

\section{The biological and cultural importance of sagu}

Previous reforms enforced throughout Siberut and greater Mentawai sought to replace sagu swamps with rice cultivation programs (Pradipta 2019), and the Mentawai people were coerced to replace sagu with 'modern' products and foods (Persoon 2001). Not only did this replacement ignore the cultural significance of sagu and displace a crucial food, building and medicinal resource (Pradipta 2019), but branded a nutritious food source as primitive and only for the poor-diminishing its status in consumers' eyes. The discouragement of sagu also failed to acknowledge the ecological efficiency of the palm for staple food production (WWF 1980; Pradipta 2019). WWF (1980) highlighted several immense ecological benefits of sagu: the palm is easily regenerated, requires no pesticide input, and provides food sources and habitat corridors for multiple species (Figure 10). Given observations of changes in rice season and harvest yields due to climate-related effects (Li et al. 2017), existing ecosystems already achieving efficient staple food production like Siberut's sagu swamps and pumonean are vital in ensuring food security for a growing population, and have considerable value toward current biodiversity conservation efforts (World Resources Institute 2019). Considering the interconnectedness of culture and biodiversity (Posey 1999, Pretty et al. 2009), those plants most valuable to a community, and the cultural traditions that ensue, are fundamental for biodiversity conservation and ecosystem regeneration.

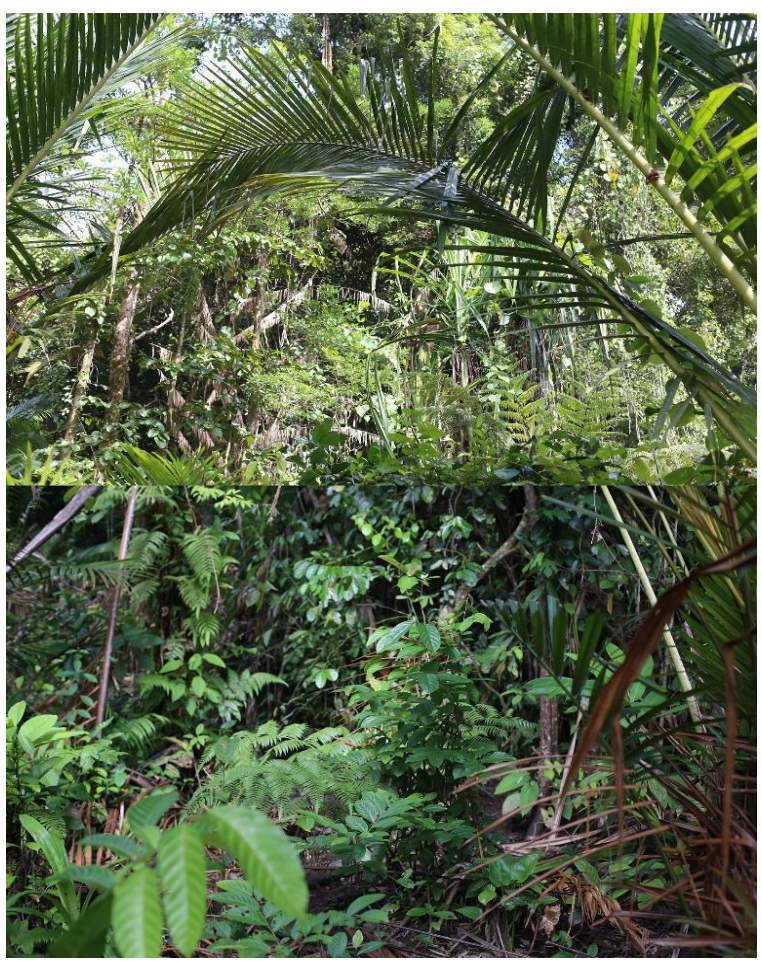

Figure 10. Examples of the diversity that may be found in sagai (Sabirut dialect) / sagu (Rereiket dialect) (Metroxylon sagu Rottb. (Arecaceae)) ecosystems.

\section{Conclusions}

Understanding the relationships between people and their biomes, and the values given to natural resources by communities are foundational to the equitable conservation of biodiversity (Hanazaki et al. 2013, Pretty et al. 2009, Sheil et al. 2005). While Mentawai's indigenous knowledge systems are globally recognised as integral to the conservation of Siberut's unique biodiversity (IPBES 2019), few studies have sought to evaluate the Mentawai community's preferences and priorities (similar to issues discussed in Sheil et al. (2005)). Given the correlation between biodiversity loss and the declining health and wellbeing of forest-based peoples (Colfer 2008, Sheil et al. 2005), recognizing and responding to local values and priorities for conservation and regeneration may have a two-fold benefit. Though representing a relatively small portion of Siberut's flora, those 63 plants that have been identified as most significant to the Sabirut and Sarereiket communities give a better perception of which plants are vital for Mentawai livelihoods, and provides a baseline from which biodiversity conservation and knowledge sharing can be guided. Perhaps more significantly, all species encountered during the study period had some use 
to the Mentawai people, demonstrating a strong relationship between Siberut's biodiversity and Mentawai TEK. Thus, the study indicates that Mentawai TEK is a rich source for understanding Siberut's ecology. Failure to consider Mentawai TEK in conservation and resource management could drive Siberut's unique biodiversity and culture into extinction. The global ecological crisis is too urgent to ignore the contributions of Mentawai TEK and local researcher input in advancing the understanding of Indonesia's ecosystems and species.

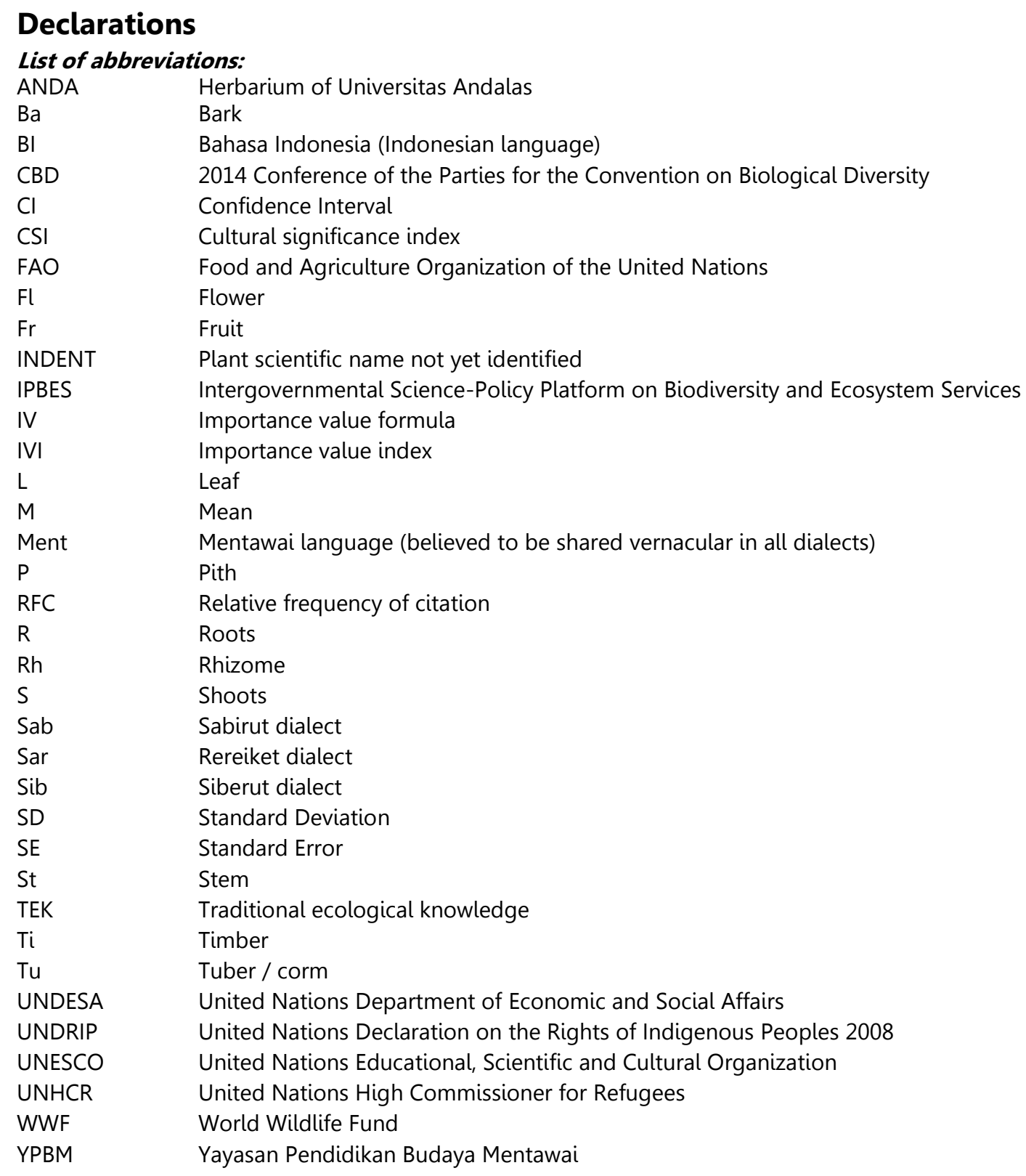

Ethics approval and consent to participate: Ethical clearance for research activities associated with this paper were approved by Institut Pertanian Bogor (IPB) Komisi Etik Penelitian yang Melibatkan Subyek Manusia, approval number: 164/IT3.KEPMSM-IPB/SK/2019, and Southern Cross University Human Research Ethics Committee, approval number: ECN-19-055. During 2016-2018, before developing the initial research proposal, informal discussions were held with staff members of YPBM, sikebbu'kat and sikerei to ask if there was a need for the proposed research, and whether they would be willing to assist in research activities. Together, these discussions highlighted the need for ethnobotanical documentation, provided the first stage of gaining consent, and directed the initial scope of the study. Before commencing the study in January 2019, procedures to inform governments and community members of the research project were followed according to both Indonesian regulation and Mentawai custom. The research outline, outcomes and community survey examples were shared with the Camat (District Leader) of Southern Siberut, requesting authorisation to carry out the proposed activities within the village districts of the Southern Siberut region. Formal written permission to undertake the study was obtained from the 
Camat, who then provided a letter of notice for the Kepala Desa (Village Leader) of each village prior to research activities commencing. Before participating in any research activities, participants read (or were read to in their preferred language) and signed an informed consent document, which outlined the research objectives, risks, benefits, and confidentiality agreements. All participants were informed they had the right to decline participation at any time throughout the research activities, and were under no obligation to reveal any knowledge they did not want to share, or felt was secret.

Consent for publication: Before participating in any research activities, participants read (or were read to by a trusted witness) and signed an informed consent document, which outlined the research objectives, risks, benefits, confidentiality agreements and consent for publication. All participants were informed they had the right to decline participation at any time throughout the research activities, and were under no obligation to reveal any knowledge they did not want to share, or considered private knowledge. Consent forms have been stored at the Yayasan Pendidikan Budaya Mentawai office, Jl. Muntei, Desa Muntei, Siberut Selatan.

Availability of data and materials: The data has not been deposited in a public repository, but has been made available to the Mentawai community through Yayasan Pendidikan Budaya Mentawai.

Competing interests: The authors have no conflict of interest, financial or otherwise.

Funding: The fieldwork activities associated with this project were funded by the National Geographic Society Early Career Research Grant (EC-53693R-19), and supported by the Australian Government Department of Foreign Affairs and Trading through the New Colombo Plan Scholarship program. These agencies were not involved in any aspect of the research preparation, data collection, nor preparation of this publication.

Authors' contributions: SML conceptualised the initial fieldwork plan and was the primary author of the manuscript, with suggestions for improvements from JDN and DL. SML and SS were involved in methodology design and implementation, and conducted the data analysis. FS was involved in fieldwork and improving the accuracy of Mentawai plant names and variations between dialects. RH offered clarification of observed socioeconomic changes. Nurainas identified plant samples at ANDA. IZS and APPH provided support in-country.

\section{Acknowledgements}

The authors are sincerely grateful to the simattaoi of both the Sabirut and Sarereiket regions, who not only participated in and supported this project but welcomed us as a part of their families and community. We would especially like to thank collaborating researchers at Yayasan Pendidikan Budaya Mentawai - Yan Mendrofa, Santi Sagari, Bastianus Ade, Filemon Sagulu, Martison Siritoitet, Raimundus Saruruk, Chindi Raflesia, Manuel Sarimau, Silvia Eka Putri, August Tonggiat - your initiative to document and protect your cultural heritage and ecological knowledge is crucial to protecting our planet, masura' bagatta. We are indebted to Sikerei Aman Masit Dere, Aman Manja and Aman Sasali. Furthermore, the depth of your knowledge of Siberut's forests is awe-inspiring, masura' bagatta.

The first author would like to express thanks to Professor Douglas Sheil for reviewing an early copy of SML's thesis submission; and to Professor Reimar Schefold for his extensive feedback on this manuscript. The first author also wishes to express deep gratitude to Rob Henry for his endless help in translating key findings and coordinating our field trips throughout Siberut. Your unwavering commitment to a more equitable future for the Mentawai people is truly admirable.

\section{Literature cited}

Alexiades MN. 1996. Selected Guidelines for Ethnobotanical Research: A Field Manual. The New York Botanical Garden, New York, USA.

Bakker L. 1999. Tiele! Turis! The social and ethnic impact of tourism in Siberut (Mentawai). MA dissertation, Leiden University.

Berkes F, Colding J, Folke C. 2000. Rediscovery of traditional ecological knowledge as adaptive management. Ecological Applications 10(5):1251-1262.

Boedhihartono AK. 2017. Can community forests be compatible with biodiversity conservation in Indonesia? Land 6(21), doi:10.3390/land6010021. 
Byg A, Balslev H. 2001. Traditional knowledge of Dypsis fibrosa (Aracaceae) in Eastern Madagascar. Economic Botany 55:263-275.

Caruso E. 2015. Conducting and Communicating Ethnobotanical Research - A Methods Manual. MedPlant, Copenhagen, Denmark.

Colfer CJP. 2008. Human Health and Forests: A Global Overview of Issues, Practice and Policy. Earthscan, Oxfordshire, UK.

Colfer CJP, Resosudarmo IAP. 2002. Which Way Forward? People, Forests and Policymaking in Indonesia. Resources for the Future, New York, USA.

Conway G. 1998. The Doubly Green Revolution: Food for All in the Twenty-First Century. Cornell University Press, New York, USA.

Corrigan C, Bingham H, Shi Y, Lewis E, Chauvenet A, Kingston N. 2018. Quantifying the contribution to biodiversity conservation of protected areas governed by indigenous peoples and local communities. Biological Conservation 227:403-412.

Corrigan C, Hay-Edie T. 2013. A toolkit to support conservation by Indigenous peoples and local communities: Building capacity and sharing knowledge for indigenous peoples' and community conserved territories and areas (ICCAs). UNEP-WCMC, Cambridge, UK.

Cunningham AB. 2001. Applied Ethnobotany: People, wild plant use and conservation. Earthscan, London, UK.

Davis W. 2009. Light at the Edge of the World. National Geographic Society, Washington D.C., USA.

Garrity DP. 2004. Agroforestry and the achievement of the Millennium Development Goals. Agroforestry Systems 61:5-17.

Gray A. 1991. Between the Spice of Life and the Melting Pot: Biodiversity Conservation and its Impact on Indigenous Peoples. International Work Group for Indigenous Affairs, Copenhagen, Denmark.

Hamilton A, Hamilton P. 2006. Plant Conservation - An Ecosystem Approach. Earthscan, London, UK.

Hanazaki N, Nakamura EM, Linder B, de Boef WS. 2013. Opportunities for ethnobotany to contribute to community biodiversity management. In Community Biodiversity Management: Promoting Resilience and the Conservation of Plant Genetic Resources, edited by WS de Boef, A Subedi and N Peroni. Routledge, New York, USA, Pp. 141-144.

Indra G, Nurainas, Chairul, Mansyurdin. 2017. Species diversity and community structure of fruit trees in Pumonean at Siberut, Mentawai Islands, Indonesia. International Journal of Current Research in Biosciences and Plant Biology 4(11):29-37.

IPBES. 2019. Summary for policymakers of the global assessment report on biodiversity and ecosystem services of the Intergovernmental Science-Policy Platform on Biodiversity and Ecosystem Services. Intergovernmental SciencePolicy Platform on Biodiversity and Ecosystem Services, Bonn, Germany.

Kumar BM, Nair PKR. 2004. The enigma of tropical homegardens. Agroforestry Systems 61:135-152.

Kusakabe K, Shrestha R, Veena N. 2015. Gender and Land Tenure in the Context of Disaster in Asia. Springer International Publishing, Cham, Switzerland.

Laurance WF, Sayer J, Cassman KG. 2014. Agricultural expansion and its impacts on tropical nature. Trends in Ecology \& Evolution 29(2):107-16.

León MC, Harvey CA. 2006. Live fences and landscape connectivity in a neotropical agricultural landscape. Agroforestry Systems 68:15-26.

Li S, Wang Q, Chun JA. 2017. Impact assessment of climate change on rice productivity in the Indochinese Peninsula using a regional-scale crop model. International Journal of Climatology 37:1147-1160.

Lin B, Chappell MJ, Vandermeer J, Smith G, Quintero E, Bezner-Kerr R, Griffith DM, Ketcham S, Latta SC, McMichael P, McGuire KL, Nigh R, Rocheleau D, Soluri J, Perfecto I. 2011. Effects of industrial agriculture on climate change and the mitigation potential of small-scale agro-ecological farms. CAB Reviews: Perspectives in Agriculture, Veterinary Science, Nutrition and Natural Resources 6(020):1-18. 
Maffi L. 2002. Endangered languages, endangered knowledge. International Social Science Journal 54(173):385393.

Martin GJ. 1995. Ethnobotany - A Methods Manual. Chapman \& Hall, London, UK.

Mazzocchi F. 2006. Western science and traditional knowledge. EMBO Reports 7(5):463-466.

McNeely JA, Schroth G. 2006. Agroforestry and biodiversity conservation - Traditional practices, present dynamics, and lessons for the future. Biodiversity and Conservation 15(2):549-554.

Minority Rights Groups International. 2017. No Escape from Discrimination: Minorities, Indigenous Peoples and the Crisis of Displacement. Minority Rights Groups International, London, UK.

Mutua J, Muriuki J, Gachie P, Bourne M, Capis J. 2014. Conservation Agriculture With Trees: Principles and Practice. World Agroforestry Centre, Nairobi, Kenya.

Nicholas G. 2018. "It's taken thousands of years, but Western science is finally catching up to Traditional Knowledge." The Conversation. Accessed 1 January 2019. https://theconversation.com/its-taken-thousands-ofyears-but-western-science-is-finally-catching-up-to-traditional-knowledge- 90291.

Nolan JM, Turner N. 2011. Ethnobotany: The Study of People-Plant Relationships. In Ethnobiology, edited by EN Anderson, D Pearsall, E Hunn and N Turner. John Wiley \& Sons, Inc, Hoboken, USA, Pp. 135-150.

Persoon G. 1998. Isolated groups or indigenous peoples; Indonesia and the international discourse. Globalization, localization and Indonesia 154 (2):281-304.

Persoon G. 2001. The management of wild and domesticated forest resources on Siberut, West Sumatra. Antropologi Indonesia 64:68-83.

Persoon G. 2008. Hidden suffering on the island of Siberut, West Sumatra. In Human Health and Forests: A Global Overview of Issues, Practice and Policy, edited by CJ Pierce Colfer. Earthscan, Oxfordshire, UK, Pp. 333-345.

Phillips O, Gentry AH. 1993. The useful plants of Tambopata, Peru: I. Statistical hypotheses tests with a new quantitative technique. Economic Botany 47:15-32.

Posey DA. 1999. Cultural and Spiritual Values of Biodiversity. United Nations Environment Programme, Nairobi, Kenya.

Pradipta L. 2019. Shifting the staple food from sago to rice: A study about food security and local communities in Mentawai Islands. Society 7(1): 37-47.

Pretty J, Adams B, Berkes F, de Athayde SF, Dudley N, Hunn E, Maffi L, Milton K, Rapport D, Robbins P, Sterling E, Stolton S, Tsing A, Vintinner E, Pilgrim S. 2009. The intersections of biological diversity and cultural diversity: Towards integration. Conservation and Society 7(2):100-112.

Quinlan M. 2005. Considerations for collecting freelists in the field: Examples from ethnobotany. Field Methods 17(3):219-234.

Retnowati E. 2003. Sustainable development through a complex agroforestry system in Indonesia. XII World Forestry Congress, Quebec City, Canada.

Reyes-García V, Guèze M, Luz AC, Paneque-Gálvez J, Macía MJ, Orta-Martínez M, Pino J, Rubio-Campillo X. 2013. Evidence of traditional knowledge loss among a contemporary indigenous society. Evolution and Human Behaviour 34(4):249-257.

Reyes-García V, Paneque-Gálvez J, Luz AC, Gueze M, Macíad MJ, Orta-Martínez M, Pino J. 2014. Cultural change and traditional ecological knowledge. An empirical analysis from the Tsimane' in the Bolivian Amazon. Human Organisation 73(2):162-173.

Rodale Institute. 2015. Regenerative Organic Agriculture and Climate Change - A Down-to-Earth Solution to Global Warming. Rodale Institute, Kutztown, USA.

Schefold R. 1989. The Origins of the Woman on the Raft: On the Prehistory of the Mentawaians. In Mentawai: Identität im Wandel auf Indonesischen Aub en Inseln, edited by W Wagner. Universitat Bremen, Bremen, Germany, Pp. 1-26. 
Sheil D, Lawrence A. 2004. Tropical biologists, local people and conservation: new opportunities for collaboration. Trends in Ecology and Evolution 19(12):634-8.

Sheil D, Puri R, Wan M, Basuki I, van Heist M, Liswanti N, Rukmiyati, Rachmatika I, Samsoedin I. 2005. Recognizing local people's priorities for tropical forest biodiversity. Ambio 35(1):17-24.

Silva VA, Andrade L, Albuquerque UP. 2006. Revising the Cultural Significance Index: The case of the Fulni-ô in Northeastern Brazil. Field Methods 18:98-108.

Sobrevila C. 2008. The Role of Indigenous Peoples in Biodiversity Conservation - The natural but often forgotten partners. The World Bank, Washington, D.C, USA.

Standley P, Hill R. 2011. Exploring the potential for Indigenous-driven tropical ethnobotany. Report of the tropical Indigenous ethnobotany centre workshop on the cultural use of plants, Cairns, 19 November 2010. CSIRO, Cairns, Australia.

State of the Tropics. 2019. State of the Tropics - Health in the Tropics. James Cook University, Townsville, Australia.

Suhandi AS, Anggraeni DE, Effendi E, Perbatakusuma EA, Meyers KJM, Wiratno. 2002. Feasiblity study of conservation concession: Reconciliatory effort between the demand of increasing local revenue and ecosystem protection in the process of power devolution - A case study from Siberut Island, Sumatra. Conservation International, Jakarta, Indonesia.

Sujarwo W, Caneva G. 2016. Using quantitative indices to evaluate the cultural importance of food and nutraceutical plants: Comparative data from the island of Bali (Indonesia). Journal of Cultural Heritage 18:342-348.

Tiwari BK, Tynsong H, Dkhar M. 2017. Traditional knowledge-based conservation and utilisation of bio-resources by War Khasi tribe of Meghalaya, India. In Knowing our Lands and Resources Indigenous and Local Knowledge and Practices related to Biodiversity and Ecosystem Services in Asia, edited by M Karki, R Hill, D Xue, W Alangui, K Ichikawa and P Bridgewater. United Nations Educational, Scientific and Cultural Organization, Paris, France, Pp. 6884.

UNDESA. 2009. State of the World's Indigenous Peoples. United Nations, New York, USA.

van Klooster C, Haabo V, and van Andel T. 2019. Our children do not have time anymore to learn about medicinal plants: How an ethnobotanical school assignment can contribute to the conservation of Saramaccan Maroon traditional knowledge. Ethnobotany Research and Applications 18:1-47.

Vogl CR, Vogl-Lukasser B, Puri RK. 2004. Tools and methods for data collection in ethnobotanical studies of homegardens. Field Methods 16(3):285-306.

Wilder BT, O'Meara C, Monti L, Nabhan G. 2016. The importance of Indigenous knowledge in curbing the loss of language and biodiversity. BioScience 66(6):499-509.

World Resources Institute. 2019. World Resources Report - Creating A Sustainable Food Future: A Menu of Solutions to Feed Nearly 10 Billion People By 2050. World Resources Institute, Washington D.C, USA.

WWF. 1980. Saving Siberut: A Conservation Master Plan. World Wildlife Fund, Bogor, Indonesia. 


\section{Appendix 1}

Table 6. Supplementary data set - Collection numbers and details of botanical samples taken during the research period.

\begin{tabular}{|c|c|c|c|c|c|c|}
\hline Scientific name & Vernacular name(s) & $\begin{array}{l}\text { Collection location (nearest } \\
\text { village) }\end{array}$ & Collector & Collection number & $\begin{array}{l}\text { Collection } \\
\text { date }\end{array}$ & Herbarium location \\
\hline INDENT & Obboi (BSib) & $\mathrm{N} / \mathrm{A}$ & $\mathrm{N} / \mathrm{A}$ & $\begin{array}{l}\text { No sample was taken for } \\
\text { this plant. During the } \\
\text { research period, Southern } \\
\text { Siberut experienced an } \\
\text { unusual drought spell where } \\
\text { it did not rain for } 5 \text { months, } \\
\text { drying out the habitat where } \\
\text { this plant is usually found. }\end{array}$ & N/A & $\mathrm{N} / \mathrm{A}$ \\
\hline Dipterocarpus fusiformis P.S.Ashton & $\begin{array}{l}\text { Elagat (BSab) / Kara-kara (BSil, BSar) / } \\
\text { Kruing (BI) }\end{array}$ & Buttui, Sothern Siberut & S Lee & SLBU001 & $4 / 8 / 19$ & UNAND Herbarium (ANDA) Padang, Indonesia \\
\hline Angiopteris evecta (G.Forst.) Hoffm. & $\begin{array}{l}\text { Bakkat laggai (BM) / Sibakkat laggai } \\
\text { (BM) }\end{array}$ & Buttui, Sothern Siberut & S Lee & SLBU002 & $4 / 8 / 19$ & UNAND Herbarium (ANDA) Padang, Indonesia \\
\hline Kibatalia arborea (Blume) G.Don & Toilat (BM) & Buttui, Sothern Siberut & S Lee & SLBU003; SLBU004 & $4 / 8 / 19$ & UNAND Herbarium (ANDA) Padang, Indonesia \\
\hline Horsfieldia sp. Blume ex DC. & Ribbu (BM) & Buttui, Sothern Siberut & S Lee & SLBU005 & $4 / 8 / 19$ & UNAND Herbarium (ANDA) Padang, Indonesia \\
\hline Justicia gendarussa Burm.f. & $\begin{array}{l}\text { Pangasele (BSar, BSil) / Pakasele } \\
\text { (BSab) }\end{array}$ & Buttui, Sothern Siberut & S Lee & SLBU006 & $5 / 8 / 19$ & UNAND Herbarium (ANDA) Padang, Indonesia \\
\hline $\begin{array}{l}\text { Cananga odoratum (Lam.) Hook.f. \& } \\
\text { Thomson }\end{array}$ & Poak (BSib) & Buttui, Sothern Siberut & S Lee & SLBU007 & $6 / 8 / 19$ & UNAND Herbarium (ANDA) Padang, Indonesia \\
\hline Amischotolype mollissima (Blume) Hassk. & Lemu-lemu (BM) & Buttui, Sothern Siberut & S Lee & SLBU008 & $6 / 8 / 19$ & UNAND Herbarium (ANDA) Padang, Indonesia \\
\hline Gleicheniaceae C.Presl & Osap (BM) & Buttui, Sothern Siberut & S Lee & SLBU020 & $10 / 11 / 19$ & UNAND Herbarium (ANDA) Padang, Indonesia \\
\hline Piper sarmentosum Roxb. & Baba (BSib) & Buttui, Sothern Siberut & S Lee & SLBU021 & 10/11/19 & UNAND Herbarium (ANDA) Padang, Indonesia \\
\hline Piper betle L. & Talingengeng (BM) / Sirih (BI) & Muntei, Southern Siberut & S Lee & SLBU022 & $10 / 11 / 19$ & UNAND Herbarium (ANDA) Padang, Indonesia \\
\hline Daemonorops angustifolia (Griff.) Mart. & Labi (BSar) / Rui (BSab) & Buttui, Sothern Siberut & S Lee & SLBU023 & $10 / 11 / 19$ & UNAND Herbarium (ANDA) Padang, Indonesia \\
\hline Etlingera elatior (Jack) R.M. Sm. & $\begin{array}{l}\text { Gojo (the whole stem) (BM) Totonan } \\
\text { (BM) / Tairatti (BM) }\end{array}$ & Buttui, Sothern Siberut & S Lee & SLBU026 & $10 / 11 / 19$ & UNAND Herbarium (ANDA) Padang, Indonesia \\
\hline Hornstedtia conica Ridl. & Kukuet (BM) & Muntei, Southern Siberut & S Lee & SLBU027 & $10 / 11 / 19$ & UNAND Herbarium (ANDA) Padang, Indonesia \\
\hline Alpinia malaccensis C.Presl & $\begin{array}{l}\text { Pasisingin (BSil, BSar) / Kasisingin } \\
\text { (BSab) / Kojo koddiai (BSib) }\end{array}$ & Buttui, Sothern Siberut & S Lee & SLBU029 & $10 / 11 / 19$ & UNAND Herbarium (ANDA) Padang, Indonesia \\
\hline Alstonia spatulata Blume & $\begin{array}{l}\text { Kakatdut (BSib) / Gite (BSil, BSar) / } \\
\text { Pulai (BI) }\end{array}$ & Matotonan, Southern Siberut & S Lee & SLMA002 & $23 / 8 / 19$ & UNAND Herbarium (ANDA) Padang, Indonesia \\
\hline Horsfieldia irya (Gaertn.) Warb. & Roat (BSab) / Doat (BSar) & Matotonan, Southern Siberut & S Lee & SLMA008 & $23 / 8 / 19$ & UNAND Herbarium (ANDA) Padang, Indonesia \\
\hline INDENT & Bolasi (BM) & Muntei, Southern Siberut & S Lee & SLMU001 & $9 / 10 / 19$ & UNAND Herbarium (ANDA) Padang, Indonesia \\
\hline INDENT & Mangguguk (BM) & Muntei, Southern Siberut & S Lee & SLMU002 & $9 / 10 / 19$ & UNAND Herbarium (ANDA) Padang, Indonesia \\
\hline Macaranga sp. Thouars & Katumanggei (BM) & Muntei, Southern Siberut & S Lee & SLMU003 & $9 / 10 / 19$ & UNAND Herbarium (ANDA) Padang, Indonesia \\
\hline Nephelium cuspidatum Blume & Babaet (BSar) / Kapa (BSab) & Muntei, Southern Siberut & S Lee & SLMU006 & $10 / 10 / 19$ & UNAND Herbarium (ANDA) Padang, Indonesia \\
\hline Shorea sp. Roxb. ex C.F.Gaertn. & Karai (BM) & Muntei, Southern Siberut & S Lee & SLMU007 & $10 / 10 / 19$ & UNAND Herbarium (ANDA) Padang, Indonesia \\
\hline
\end{tabular}




\begin{tabular}{|c|c|c|c|c|c|c|}
\hline Erythrina subumbrans (Hassk.) Merr. & Mancuat (BM) / Matcuat (BM) & Muntei, Southern Siberut & S Lee & SLMU008 & 10/10/19 & UNAND Herbarium (ANDA) Padang, Indonesia \\
\hline Ludwigia linifolia Poir. & $\begin{array}{l}\text { Mumru tuktuk (BSar) / Lottek-lottek } \\
\text { (BSab) }\end{array}$ & Muntei, Southern Siberut & S Lee & SLMU009; SLMU010 & $11 / 10 / 19$ & UNAND Herbarium (ANDA) Padang, Indonesia \\
\hline $\begin{array}{l}\text { Lansium parasiticum (Osbeck) K.C.Sahni \& } \\
\text { Bennet }\end{array}$ & $\begin{array}{l}\text { Selak (BSab) / Tiat (BSar, BSil) / } \\
\text { Siamung (BSar, BSil) }\end{array}$ & Muntei, Southern Siberut & S Lee & SLMU011 & $11 / 10 / 19$ & UNAND Herbarium (ANDA) Padang, Indonesia \\
\hline Durio spp. Adans. & Doriat (BM) / Dorian (BSil) & Muntei, Southern Siberut & S Lee & SLMU012 & $11 / 10 / 19$ & UNAND Herbarium (ANDA) Padang, Indonesia \\
\hline Hibiscus rosa-sinensis $\mathrm{L}$. & Bekeu (BM) / Kembang sepatu (BI) & Muntei, Southern Siberut & S Lee & SLMU013 & $11 / 10 / 19$ & UNAND Herbarium (ANDA) Padang, Indonesia \\
\hline Nephelium lappaceum $\mathrm{L}$. & $\begin{array}{l}\text { Gairabbik (BSab, BSar) / Rambutan } \\
\text { (BI) }\end{array}$ & Muntei, Southern Siberut & S Lee & SLMU014 & $11 / 10 / 19$ & UNAND Herbarium (ANDA) Padang, Indonesia \\
\hline Graptophyllum pictum (L.) Griff. & Aileleppet (BM) & Muntei, Southern Siberut & S Lee & SLMU015 & $11 / 10 / 19$ & UNAND Herbarium (ANDA) Padang, Indonesia \\
\hline Cymbopogon citratus (DC.) Stapf & $\begin{array}{l}\text { Siat-siat (BSab) / Jiat-jiat (BSar) / } \\
\text { Serai (BI) }\end{array}$ & Muntei, Southern Siberut & S Lee & SLMU016 & $11 / 10 / 19$ & UNAND Herbarium (ANDA) Padang, Indonesia \\
\hline Rottboellia sp. Naezén & Boku-bokuk (BM) & Muntei, Southern Siberut & S Lee & SLMU017 & $11 / 10 / 19$ & UNAND Herbarium (ANDA) Padang, Indonesia \\
\hline Barringtonia racemosa (L.) Spreng. & Ngirip (BSib) / Irip (BSib) & Muntei, Southern Siberut & S Lee & SLMU018 & $11 / 10 / 19$ & UNAND Herbarium (ANDA) Padang, Indonesia \\
\hline Bamboo types & Manggeak (BM) / Bambu (BI) & Muntei, Southern Siberut & S Lee & SLMU019 & $4 / 8 / 19$ & UNAND Herbarium (ANDA) Padang, Indonesia \\
\hline Curcuma longa L. & Kiniu (BM) / Kunyit (BI) & Muntei, Southern Siberut & S Lee & SLMU020 & $11 / 10 / 19$ & UNAND Herbarium (ANDA) Padang, Indonesia \\
\hline Acorus calamus $\mathrm{L}$. & $\begin{array}{l}\text { Sumamra (BSar, BSil) / Butek-butek } \\
\text { (BSab) }\end{array}$ & Muntei, Southern Siberut & S Lee & SLMU021 & $11 / 10 / 19$ & UNAND Herbarium (ANDA) Padang, Indonesia \\
\hline Psidium guajava L. & $\begin{array}{l}\text { Jabbui (BM) / Sabbui (BSar) Jambu } \\
\text { biji (BU) }\end{array}$ & Muntei, Southern Siberut & S Lee & SLMU022 & $11 / 10 / 19$ & UNAND Herbarium (ANDA) Padang, Indonesia \\
\hline Alpinia galangal (L.) Willd. & Oroket (BSab) / Palakkokoai (BSar) & Muntei, Southern Siberut & S Lee & SLMU023; SLMU024 & $11 / 10 / 19$ & UNAND Herbarium (ANDA) Padang, Indonesia \\
\hline Kaempferia galangal L. & $\begin{array}{l}\text { Kopuk (BSab) / Sikopuk (BSar) / } \\
\text { Kencur (BI) }\end{array}$ & Muntei, Southern Siberut & S Lee & SLMU026 & $11 / 10 / 19$ & UNAND Herbarium (ANDA) Padang, Indonesia \\
\hline Cordyline fruticosa (L.) A.Chev. & Boblo (BM) & Muntei, Southern Siberut & S Lee & SLMU027; SLMU028 & $11 / 10 / 19$ & UNAND Herbarium (ANDA) Padang, Indonesia \\
\hline $\begin{array}{l}\text { Codiaeum variegatum (L.) Rumph. ex } \\
\text { A.Juss. }\end{array}$ & Surak (BM) & Muntei, Southern Siberut & S Lee & $\begin{array}{l}\text { SLMU029; SLMU030; } \\
\text { SLMU031; SLMU032 }\end{array}$ & $11 / 10 / 19$ & UNAND Herbarium (ANDA) Padang, Indonesia \\
\hline Mussaenda frondosa $\mathrm{L}$. & Mumunen (BSib) & Muntei, Southern Siberut & S Lee & SLMU033 & $11 / 10 / 19$ & UNAND Herbarium (ANDA) Padang, Indonesia \\
\hline Musa spp. L. & $\begin{array}{l}\text { Magok (BSar, BSil) / Bagok (BSab) / } \\
\text { Pisang (BI) }\end{array}$ & Muntei, Southern Siberut & S Lee & SLMU034 & $22 / 11 / 19$ & UNAND Herbarium (ANDA) Padang, Indonesia \\
\hline Manihot sp. Mill. & $\begin{array}{l}\text { Bairatti (BSab) / Sipukaju (BSil, BSar) / } \\
\text { Ubi kayu (BI) }\end{array}$ & Muntei, Southern Siberut & S Lee & SLMU035 & $22 / 11 / 19$ & UNAND Herbarium (ANDA) Padang, Indonesia \\
\hline Ipomoea sp. L. & $\begin{array}{l}\text { Siputeteket (BSil, BSar) / Gobik (BSab) } \\
\text { / Ubi jalar (BI) }\end{array}$ & Muntei, Southern Siberut & S Lee & SLMU036 & $22 / 11 / 19$ & UNAND Herbarium (ANDA) Padang, Indonesia \\
\hline $\begin{array}{l}\text { Hedychium coronarium J.Koenig / } \\
\text { Hedychium flavescens Carey ex Roscoe }\end{array}$ & $\begin{array}{l}\text { Kakainauk (BSab) / Simakainauk } \\
\text { (BSar, BSil) }\end{array}$ & Muntei, Southern Siberut & S Lee & SLMU037 & $22 / 11 / 19$ & UNAND Herbarium (ANDA) Padang, Indonesia \\
\hline Melastoma sylvaticum Blume & $\begin{array}{l}\text { Sibekak (BM) / Sibetu (BSab) / Eruk } \\
\text { teinung (BSil, BSar) }\end{array}$ & Muntei, Southern Siberut & S Lee & SLMU038 & $22 / 11 / 19$ & UNAND Herbarium (ANDA) Padang, Indonesia \\
\hline Macaranga tanarius (L.) Müll.Arg. & Ottot (BM) / Pari-pari (BSib) & Muntei, Southern Siberut & S Lee & SLMU039 & $22 / 11 / 19$ & UNAND Herbarium (ANDA) Padang, Indonesia \\
\hline Colocasia esculenta (L.) Schott & Gettek (BM) / Talas (BI) / Keladi (BI) & Muntei, Southern Siberut & S Lee & SLMU040 & $22 / 11 / 19$ & UNAND Herbarium (ANDA) Padang, Indonesia \\
\hline Curcuma zanthorrhiza Roxb. & Kiniu sailuk (BM) / Temulawak (BI) & Muntei, Southern Siberut & S Lee & SLMU041 & $22 / 11 / 19$ & UNAND Herbarium (ANDA) Padang, Indonesia \\
\hline Cocos nucifera L. & Toitet (BM) / Kelapa (BI) & Muntei, Southern Siberut & S Lee & SLMU043 & $22 / 11 / 19$ & UNAND Herbarium (ANDA) Padang, Indonesia \\
\hline Arenga pinnata (Wurmb.) Merr. & Poula (BM) / Katsaila (BM) & Muntei, Southern Siberut & S Lee & SLMU044 & $22 / 11 / 19$ & UNAND Herbarium (ANDA) Padang, Indonesia \\
\hline Rhizophora apiculata Blume & Bakat (BM) / Bakau (BI) & Muntei, Southern Siberut & S Lee & SLMU045; SLMU046 & $22 / 11 / 19$ & UNAND Herbarium (ANDA) Padang, Indonesia \\
\hline Eleusine indica (L.) Gaertn. & $\begin{array}{l}\begin{array}{l}\text { Ngiti-ngitit (BSib) / Komensakkokok } \\
\text { (BSab) }\end{array} \\
\end{array}$ & Muntei, Southern Siberut & S Lee & SLMU047 & $22 / 11 / 19$ & UNAND Herbarium (ANDA) Padang, Indonesia \\
\hline
\end{tabular}




\section{Ethnobotany Research and Applications}

\begin{tabular}{|c|c|c|c|c|c|c|}
\hline Zingiber officinale Roscoe & Laigak (BM) / Jahe (Bl) & Muntei, Southern Siberut & S Lee & SLMU048 & $22 / 11 / 19$ & UNAND Herbarium (ANDA) Padang, Indonesia \\
\hline Calamus caesius Blume & Mandorau (BM) & Buttui, Sothern Siberut & S Sagari & SSBU001 & $4 / 8 / 19$ & UNAND Herbarium (ANDA) Padang, Indonesia \\
\hline Shorea pauciflora King & Katuka (BM) & Buttui, Sothern Siberut & S Sagari & SSBU002 & $4 / 8 / 19$ & UNAND Herbarium (ANDA) Padang, Indonesia \\
\hline Pentace triptera Mast. & Kaboi (BSib) & Buttui, Sothern Siberut & S Sagari & SSBU003 & $4 / 8 / 19$ & UNAND Herbarium (ANDA) Padang, Indonesia \\
\hline Hopea dryobalanoides Miq. & Mancemi (BM) & Buttui, Sothern Siberut & S Sagari & SSBU004 & $4 / 8 / 19$ & UNAND Herbarium (ANDA) Padang, Indonesia \\
\hline Gymnacranthera sp. (A.DC.) Warb. & Ngalou pataddekat (BSar) & Buttui, Sothern Siberut & S Sagari & SSBU005 & $4 / 8 / 19$ & UNAND Herbarium (ANDA) Padang, Indonesia \\
\hline $\begin{array}{l}\text { Goniothalamus sp. (Blume) Hook.f \& } \\
\text { Thomson }\end{array}$ & Simuinek (BSar) & Buttui, Sothern Siberut & S Sagari & SSBU006 & $4 / 8 / 19$ & UNAND Herbarium (ANDA) Padang, Indonesia \\
\hline Calamus sp. L. & Pelege (BM) & Buttui, Sothern Siberut & S Sagari & SSBU020; SSBU022 & $13 / 11 / 19$ & UNAND Herbarium (ANDA) Padang, Indonesia \\
\hline Daemonorops sp. Blume & Sasa (BM) & Buttui, Sothern Siberut & S Sagari & SSBU021; SSBU023 & $13 / 11 / 19$ & UNAND Herbarium (ANDA) Padang, Indonesia \\
\hline $\begin{array}{l}\text { Etlingera coccinea (Blume) S.Sakai \& } \\
\text { Nagam. }\end{array}$ & Pelekak (BSib) & Buttui, Sothern Siberut & S Sagari & SSBU024 & $13 / 11 / 19$ & UNAND Herbarium (ANDA) Padang, Indonesia \\
\hline Metroxylon sagu Rottb. & Sagu (BSar, BSil) / Sagai (BSab) & Buttui, Sothern Siberut & S Sagari & SSBU026 & $13 / 11 / 19$ & UNAND Herbarium (ANDA) Padang, Indonesia \\
\hline
\end{tabular}

